\title{
Isolation, Identification and Antibiotic Susceptibility of pathogenic Bacteria Isolated from Clinical Samples
}

\author{
Ehsan F. Hussein* \\ *University of Summer / College of Basic Education / Department of Science
}

\begin{abstract}
Samples have been collected, through the period from January/2016 to June/2016. This for isolation and identification of pathogenic gram negative bacteria. The samples were involve 50 burns samples for isolate Pseudomonas aeruginosa, 50 stool samples for isolate Escherichia coli and 50 urine samples for isolate Enterobacter cloacae and Proteus mirabilis. The isolates were identified by ten biochemical tests as well as the sensitivity and resistant were tested by thirteen type of antibiotics. These antibiotics explained different mode of action in their activity, were resistant, moderate and sensitive, some of them have significant differences when comparison together and some have no. The Trimethoprim, Amikacin, Aztreonam and Novobiocin have high activity against Ps. aeruginosa, E. coli, E. cloacae and P. mirabilis respectively, as well as the Aztreonam was more active when comparison the activity of these antibiotics together.
\end{abstract}

Keyword: Pathogenic gram negative bacteria, Resistant, Antibiotic, biochemical tests.

\section{Introduction}

Gram negative bacteria play important role in infectious diseases, but the Pseudomonas aeruginosa, Escherichia coli, Enterobacter cloacae and Proteus mirabilis now become the broad spectrum species involved in nosocomial infections (Anuj Nautiyal et al., 2015).Antibiotics identified, action modes and bacterial resistance mechanisms have been researched topics in academia until recently in the pharmaceutical applications (Davies and Davies., 2010). Bacteria can be resistant one or more type of antibiotics, the mechanisms by which bacteria resist antibiotics include, alter the receptor for the antibiotic, decrease antibiotic amount that reaches the receptor by preventing entry or removal, antibiotic alteration or destroying and develop resistant metabolic pathways (Fisher and Mobashery., 2010).

The Pseudomonas aeruginosa are aerobic, motile gram negative rod bacteria worldwide distributed and found as skin flora and in the majority of environmental sources (Jabalameli et al., 2011). It is can survive in different environments such as aquatic and terrestrial and contributes strongly to nosocomial infections and infected of immunocompromised peoples (Eguchi et al., 2013). This bacteria has widespread antibiotic resistance among their clinical isolates, as well as considered as one of the most important bacterial agents associated with nosocomial infections (Mirsalehian et al., 2010), also known as the most common bacterial agents in the burn patients (Azimi et al., 2015). This bacteria causes invasive infections in burn and this lead to sepsis from invasive burn wound infection (Alhazmi., 2015).

The inherent antibiotics resistance mechanisms for Pseudomonas aeruginosa involve lower outer membrane permeability, increased expression of efflux pumps for different specificity and presence betalactamase give this bacteria resistance mechanisms to commonly used antibiotics (Strateva and Yordanov 2009). This is true for several opportunistic pathogenic bacteria like Ps. aeruginosa which causes number of chronic infections due to resistant to modern antimicrobial therapies (Gellatly and Hancock 2013). Whether in the clinic or in the natural environment this bacteria encounters multiple classes of traditional antibiotics, antiseptics and disinfectants (Murray et al., 2015).

The Escherichia coli are a microorganism that commonly live in the large intestine of humans and other warm blooded animals, this is the most prevalent facultative gram negative bacteria in the human fecal flora and usually inhabit the colon as commensal microorganism (Manikandan and Amsath., 2014). Some strains of this bacteria can cause disease inside and outside of the gastrointestinal tract (Manikandan and Amsath., 2014). This bacteria is used in the variety of fields in both the industrial and medical, also the most used bacterium in the technology of recombinant DNA (Yoo et al., 2009).

E. coli is has resistant to therapeutic levels of the first $\beta$-lactam that act on cell wall, and this because of its outer membrane is barrier to this type of antibiotics (Allocati et al., 2013). Also, this bacteria have resistant to several different types of antibiotics with distinct mechanisms of action (Johnson et al., 2012). The emergence and spread of resistant against multidrug for strains of Escherichia coli is complicating and become difficult in the treatment of several serious infections caused by this bacteria (Allocati et al., 2013). Family of Enterobacteriaceae, specillay of Escherichia coli. are the most frequent cause of hospital and this lead to community acquired infections (Pitout., 2012). 
The use of antimicrobial agents in wrong ways for animals and humans treatment lead to the emergence and diffusion of the several antibiotic resistant strains of Escherichia coli that also capable to infect other humans through either direct contact with animals or through consumption of contaminated food with this bacteria (Ewers at al., 2012). Escherichia coli have ability to survive and adapt in different extra intestinal locations and to produce resistances between humans, animals and their products through environment by several transmission pathways (Ewers at al., 2012).

The Enterobacter belonging to the family of Enterobacteriaceae which is common Gram negative, rod shaped and non spore forming bacteria, live as facultative anaerobic and have been reported as important opportunistic and multi resistant bacterial pathogens and causes infection for humans (Davin-Regli and Pages., 2015). There are two main known species for this genus, which are E. aerogenes and E. cloacae have take non clinical significance as opportunistic bacteria and have emerged as nosocomial pathogens (Mezzatesta et al., 2012). Enterobacter cloacae now is the most frequently observed in clinical isolate among Enterobacter species but, the pathogenicity and factors contributing in the disease caused by this bacteria still not understood (DavinRegli and Pages., 2015). This bacteria has been associations with clinical samples particular in urines with property of antibiotics resistant (Pestourie et al.,2014). E. cloacae has several mechanisms for resistant of wide range of antibiotics (Davin-Regli and Pages., 2015). Various studies on antibiotic resistance E .cloacae which associated with low level susceptibility to several antibiotics by inducing the over expression of the efflux pump (Perez et al., 2012).

The Proteus is genus belonging to the family of Enterobactericeae and of gram negative bacteria, this is widespread in the environment and makes third as the cause of hospital acquired infections it frequently causes nosocomial infections of the urinary tract (Bahashwan., 2013). This genus consists of four species: Proteus vulgaris, Proteus penneri, Proteus myxofaciens and Proteus mirabilis (Penner et.al., 1984). The latter is the most causative agent of majority Proteus infections (Feglo et al., 2010) and the most one of bacteria that responsible for urinary tract infections (Nabeela et al., 2004).

Proteus mirabilis is generally susceptible to most antibiotics, including beta-lactams, aminoglycosides and imipenem but it has intrinsic resistance to nitrofurantoin, tetracycline and colistin (Hara et al., 2000). However, the multidrug resistant strains of Proteus species has also been reported worldwide (Singla et al., 2015). As well as has ability to resistant to several different types of antibiotics and called multi antibiotics resistant (Dadheech et al., 2015). In addition to including recent evidences of multidrug resistance $P$. mirabilis (Wong et al., 2013).

\section{Isolation of pathogenic gram negative bacterial:}

\section{Materials and Methods}

Table:1. Percentage of all isolated

\begin{tabular}{|l|l|l|l|l|}
\hline Bacterial type & Site of Collection & Number & No. of isolates & Samples (\%) \\
\hline Ps. aeruginosa & Burns & 50 & 22 & 30.137 \\
\hline E. coli & Stool & 50 & 18 & 24.657 \\
\hline E. cloacae & & & 15 & 20.548 \\
\cline { 1 - 4 } P. mirabilis & Urine & 50 & 18 & 24.657 \\
\hline Total & All isolates 48.667 \% & 150 & 73 & 99.999 \\
\hline
\end{tabular}

This table showed the percent of all isolated was 48.667, as well as Ps. aeruginosa isolated from burn, E. coli from Stool, E. cloacae from Urine and P. mirabilis from Urine were 30.137, 24.657, 20.548 and 24.657 respectively.

\section{Identification of isolates:}

Table:2. Identification of pathogenic bacteria by ten biochemical tests

\begin{tabular}{|c|c|c|c|c|c|c|c|c|c|c|}
\hline Bacterial & Gram stain & Indol & Urease & Oxidase & Catalase & Citrate & MR & VP & $\mathrm{H} 2 \mathrm{~S}$ & Gas \\
\hline Ps. aeruginosa* & ( &. & & + & + & + & 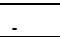 &. & - & \\
\hline E. coli** & . & + & - & . & + & . & + & - & - & + \\
\hline E. cloacae $* * * *$ & & & $\pi$ & - & + & + & - & + & . & + \\
\hline P. mirabilis $* * * * *$ & & 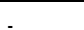 & + & 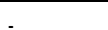 & + & + & + & + & + & s \\
\hline
\end{tabular}

This table explained the results of biochemical tests for identified isolates after growing on three plates, were Nutrient agar, Mac Conkey's agar and Blood agar. These bacteria were identified by biochemical tests according to the Society of American Bacteriologists (Pelczar et al., 1957). The (+) refer to positive and (-) to negtive (*Cheesbrough., 2006 ; **Otoikhian \& Tanimowo 2016 ; ***Stiles \& LAI., 1981 and ****Holt et al., 1994) 
Identification of antibiotics sensitivity and resistant for bacterial samples:.

Antibiotic susceptibility test:

Antibiotic sensitivity tests for these bacteria were carried out by disc diffusion technique on Muller Hinton agar plates (Bauer et al., 1966).

\section{Statistical analysis:}

The statistical analysis was performed with the Statistical Package for Social Sciences (SPSS) version 20 for calculate Mean, Medium Std. Deviation and Std. Error of Mean as well as the significance between antibiotics according to One-Way ANOVA by descriptive exclude cases analysis by analysis with LSD at $95 \%$ confidence and significant level $(\mathrm{P}=0.05)$

\section{Results}

One hundred and fifty Samples have been collected, through the period from January/2016 to June/2016. This for isolation and identification of pathogenic gram negative bacteria. The samples were involve 50 burns samples for isolate Pseudomonas aeruginosa, 50 stool samples for isolate Escherichia coli and 50 urine samples for isolate Enterobacter cloacae and Proteus mirabilis.

Figure:1. The inhibition zones formed by different types of antibiotics

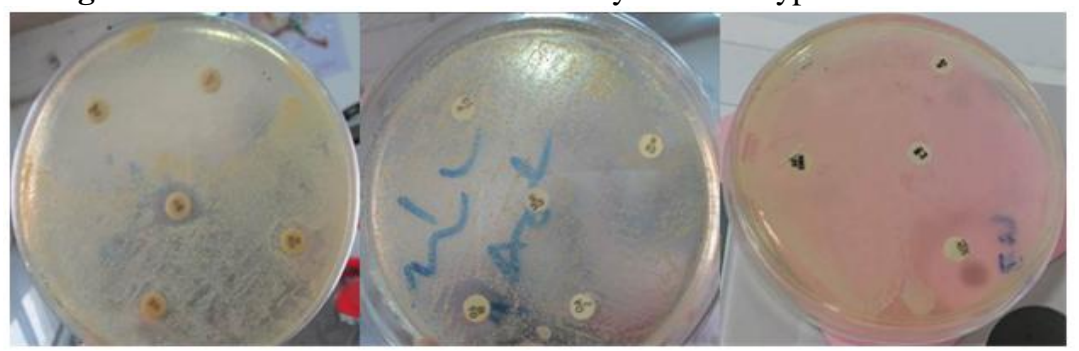

Table:3. Show the replicates of inhibition zones formed by different types of antibiotics against Ps. Aeruginosa

\begin{tabular}{|c|c|c|c|c|c|c|c|c|c|c|c|c|c|}
\hline \multicolumn{14}{|c|}{ Replicates of Inhibition zones measured by (mm) } \\
\hline Replicates/* & AX $15 \mu \mathrm{g}$ & $\begin{array}{r}\text { AK } \\
30 \\
\mu \mathrm{g}\end{array}$ & $\begin{array}{ll}\text { TMPP } & 10 \\
& \mu \mathrm{g}\end{array}$ & $\begin{array}{ll}\text { ATM } & 30 \\
& \mu \mathrm{g}\end{array}$ & $\begin{array}{ll}\text { SMZ } & 25 \\
& \mu \mathrm{g}\end{array}$ & $\begin{array}{rr}\text { PY } & 100 \\
& \mu \mathrm{g}\end{array}$ & $\begin{array}{r}\text { NV } 30 \\
\mu \mathrm{g}\end{array}$ & $\begin{array}{ll}\text { E } & 15 \\
& \mu \mathrm{g}\end{array}$ & $\begin{array}{ll}\text { R } & 40 \\
& \mu \mathrm{g}\end{array}$ & $\begin{array}{ll}P & 10 \\
\mu \mathrm{g}\end{array}$ & $\begin{array}{r}\mathrm{OA} 2 \\
\mu \mathrm{g}\end{array}$ & $\begin{aligned} 10 \\
\mu \mathrm{g}\end{aligned}$ & $\begin{array}{r}\mathrm{CC} 5 \\
\mu \mathrm{g}\end{array}$ \\
\hline 1 & 7 & 6 & 10 & 12 & 0 & 0 & 10 & 5 & 4 & 1 & 0 & 18 & 10 \\
\hline 2 & 5 & 10 & 9 & 0 & 4 & 3 & 7 & 6 & 0 & 6 & 2 & 0 & 0 \\
\hline 3 & 10 & 5 & 7 & 6 & 0 & 0 & 6 & 9 & 0 & 2 & 0 & 5 & 6 \\
\hline 4 & 5 & 0 & 13 & 0 & 3 & 5 & 8 & 15 & 8 & 5 & 0 & 11 & 3 \\
\hline 5 & 12 & 0 & 5 & 5 & 0 & 0 & 15 & 8 & 5 & 4 & 3 & 5 & 0 \\
\hline 6 & 7 & 18 & 12 & 6 & 5 & 5 & 7 & 6 & 5 & 0 & 4 & 0 & 4 \\
\hline 7 & 4 & 5 & 7 & 0 & 2 & 7 & 13 & 3 & 2 & 6 & 4 & 10 & 4 \\
\hline 8 & 5 & 2 & 11 & 12 & 3 & 9 & 5 & 12 & 5 & 3 & 8 & 4 & 1 \\
\hline 9 & 10 & 4 & 15 & 10 & 10 & 6 & 14 & 9 & 9 & 6 & 6 & 6 & 5 \\
\hline 10 & 8 & 5 & 5 & 2 & 7 & 5 & 6 & 7 & 5 & 7 & 5 & 5 & 0 \\
\hline 11 & 9 & 9 & 15 & 8 & 5 & 7 & 12 & 9 & 7 & 4 & 6 & 7 & 6 \\
\hline 12 & 17 & 7 & 15 & 0 & 0 & 5 & 9 & 0 & 3 & 0 & 8 & 6 & 8 \\
\hline 13 & 10 & 8 & 7 & 16 & 13 & 10 & 10 & 18 & 10 & 10 & 4 & 8 & 4 \\
\hline 14 & 0 & 0 & 8 & 6 & 8 & 7 & 8 & 7 & 0 & 8 & 9 & 5 & 1 \\
\hline 15 & 0 & 10 & 19 & 0 & 7 & 8 & 7 & 11 & 7 & 3 & 3 & 7 & 3 \\
\hline Mean & 7.266 & 5.933 & 10.533 & 5.533 & 4.466 & 5.134 & 9.133 & 8.333 & 4.667 & 4.333 & 4.133 & 6.466 & 3.667 \\
\hline Median & 7.000 & 5.000 & 10.000 & 6.000 & 4.000 & 5.000 & 8.000 & 8.000 & 5.000 & 4.000 & 4.000 & 6.000 & 4.000 \\
\hline Std. Deviation & 4.431 & 4.787 & 4.206 & 5.249 & 3.961 & 3.181 & 3.113 & 4.498 & 3.221 & 2.919 & 2.924 & 4.374 & 3.015 \\
\hline $\begin{array}{r}\text { Std. Error of } \\
\text { Mean }\end{array}$ & 1.144 & 1.236 & 1.086 & 1.355 & 1.022 & 0.821 & 0.803 & 1.161 & 0.821 & 0.753 & 0.756 & 1.129 & 0.779 \\
\hline
\end{tabular}

This table explained the Mean, Median, Std. deviation and Std. error for replicates of inhibition zones that measured by $(\mathrm{mm})$ for each antibiotics against Ps. aeruginosa bacteria. And showed the (TMP 10) antibiotic has high activity $(10.533 \mathrm{~mm})$ while the (CC 5) antibiotic has low activity $(3.667 \mathrm{~mm})$ against Ps. aeruginosa bacteria by mean measured.

\begin{tabular}{|c|c|c|c|}
\hline *AX 15 & Amoxicillin15 $\mu \mathrm{g}$ & ${ }^{*}$ E 15 & Erythromycin $15 \mu \mathrm{g}$ \\
\hline *AK 30 & Amikacin30 $\mu \mathrm{g}$ & ${ }^{*} \mathrm{R} 40$ & Rifaximin40 $\mu \mathrm{g}$ \\
\hline${ }^{*}$ TMP 10 & Trimethoprim10 $\mu \mathrm{g}$ & ${ }^{*} \mathbf{P} 10$ & Penicillin-G10 $\mu \mathrm{g}$ \\
\hline${ }^{*}$ ATM 30 & Aztreonam30 $\mu \mathrm{g}$ & *OA 2 & Oxolinic acid2 $\mu \mathrm{g}$ \\
\hline${ }^{*}$ SMZ 25 & Sulfamethoxazole25 $\mu \mathrm{g}$ & ${ }^{*}$ B 10 & Bacitracin10 $\mu \mathrm{g}$ \\
\hline *PY 100 & Carbnicillin100 $\mu \mathrm{g}$ & ${ }^{*} \mathrm{CC} 5$ & Clindamycin5 $\mu \mathrm{g}$ \\
\hline
\end{tabular}


Isolation, Identification and Antibiotic Susceptibility of pathogenic Bacteria Isolated from Clinical..

Table:4. Show the significantly study according to LSD system for different types of antibiotics against Ps. Aeruginosa

\begin{tabular}{|c|c|c|c|c|c|c|c|c|c|c|c|c|c|c|}
\hline \multicolumn{15}{|c|}{ Significantly study according to LSD at P-Value $=0.05$} \\
\hline $\begin{array}{l}\text { Y sid } \\
\text { antibi }\end{array}$ & $\begin{array}{ll}\text { s) } & \\
\text { s) side }\end{array}$ & $\begin{array}{l}\text { AXl5 } \\
\mu \mathrm{g}\end{array}$ & $\begin{array}{l}\text { AK30 } \\
\mu \mathrm{g}\end{array}$ & $\begin{array}{l}\text { TMP10 } \\
\mu \mathrm{g}\end{array}$ & $\begin{array}{l}\text { ATM30 } \\
\mu \mathrm{g}\end{array}$ & $\begin{array}{l}\text { SMZ25 } \\
\mu \mathrm{g}\end{array}$ & $\begin{array}{l}\text { PY100 } \\
\mu \mathrm{g}\end{array}$ & $\begin{array}{l}\text { NV30 } \\
\mu g\end{array}$ & $\begin{array}{l}\text { E } 15 \\
\mu \mathrm{g}\end{array}$ & $\begin{array}{l}\mathrm{R} 40 \\
\mu \mathrm{g}\end{array}$ & $\begin{array}{ll} & 10 \\
\mu g & \end{array}$ & $\begin{array}{l}0 A 2 \\
\mu \mathrm{g}\end{array}$ & $\begin{array}{l}\text { B } 10 \\
\mu \mathrm{g}\end{array}$ & $\begin{array}{l}\mathrm{CC} 5 \\
\mu \mathrm{g} \\
\end{array}$ \\
\hline \multirow{2}{*}{$\begin{array}{l}\mathrm{AX} \\
15 \mu \mathrm{g}\end{array}$} & Sig. & & 0.352 & $0.002 *$ & 0.227 & 0.052 & 0.137 & 0.193 & 0.457 & 0.071 & $0.042^{*}$ & $0.031^{*}$ & 0.576 & $0.013^{*}$ \\
\hline & M.D. ** & & -1.333 & 4.601 & -1.733 & -2.801 & -2.133 & 1.866 & 1.066 & -2.601 & -2.933 & -3.133 & -0.801 & -3.601 \\
\hline \multirow{2}{*}{$\begin{array}{l}\mathrm{AK} \\
30 \mu \mathrm{g}\end{array}$} & Sig. & 0.352 & & $0.002^{*}$ & 0.780 & 0.306 & 0.576 & $0.026^{*}$ & 0.095 & 0.377 & 0.264 & 0.210 & 0.710 & 0.115 \\
\hline & M.D. ** & 1.333 & & 4.601 & -0.401 & -1.466 & -0.801 & 3.201 & 2.401 & -1.266 & -1.601 & -1.801 & 0.533 & -2.266 \\
\hline \multirow{2}{*}{$\begin{array}{l}\text { TMP } \\
10 \mu \mathrm{g}\end{array}$} & Sig. & $0.023^{*}$ & $0.002 *$ & & $0.001^{*}$ & $0.001^{*}$ & $0.001^{*}$ & 0.329 & 0.126 & $0.001^{*}$ & $0.001^{*}$ & $0.001^{*}$ & $0.005^{*}$ & $0.001^{*}$ \\
\hline & M.D. ${ }^{* *}$ & -3.266 & -4.601 & & -5.001 & -6.066 & -5.401 & -1.401 & -2.201 & -5.866 & -6.201 & -6.401 & -4.066 & -6.866 \\
\hline \multirow{2}{*}{$\begin{array}{l}\text { ATM } \\
30 \mu \mathrm{g}\end{array}$} & Sig. & 0.227 & 0.780 & $0.001^{*}$ & & 0.457 & 0.780 & $0.013^{*}$ & 0.052 & 0.545 & 0.402 & 0.329 & 0.515 & 0.193 \\
\hline & M.D. ${ }^{* *}$ & 1.733 & 0.401 & 5.001 & & -1.066 & -0.401 & 3.601 & 2.801 & -0.866 & -1.201 & -1.401 & 0.933 & -1.866 \\
\hline \multirow{2}{*}{$\begin{array}{l}\text { SMZ } \\
25 \mu \mathrm{g}\end{array}$} & Sig. & 0.052 & 0.306 & $0.001^{*}$ & 0.457 & & 0.642 & $0.001^{*}$ & $0.007^{*}$ & 0.889 & 0.926 & 0.816 & 0.163 & 0.576 \\
\hline & M.D. ${ }^{* *}$ & 2.801 & 1.466 & 6.066 & 1.066 & & 0.666 & 4.666 & 3.866 & 0.201 & -0.133 & -0.333 & 2.001 & -0.801 \\
\hline \multirow{2}{*}{$\begin{array}{l}\text { PY } \\
100 \mu \mathrm{g}\end{array}$} & Sig. & 0.137 & 0.576 & $0.001^{*}$ & 0.780 & 0.642 & & $0.006^{*}$ & $0.026^{*}$ & 0.744 & 0.576 & 0.485 & 0.352 & 0.306 \\
\hline & M.D. ** & 2.133 & 0.801 & 5.401 & 0.401 & -0.666 & & 4.001 & 3.201 & -0.466 & -0.801 & -1.001 & 1.333 & -1.466 \\
\hline \multirow{2}{*}{$\begin{array}{l}\mathrm{NV} \\
30 \mu \mathrm{g}\end{array}$} & Sig. & 0.193 & $0.026^{*}$ & 0.329 & $0.013^{*}$ & $0.001^{*}$ & $0.006^{*}$ & & 0.576 & $0.002^{*}$ & $0.001^{*}$ & $0.001^{*}$ & 0.064 & $0.001^{*}$ \\
\hline & M.D. ${ }^{* *}$ & -1.866 & -3.201 & 1.401 & -3.601 & -4.666 & -4.001 & & -0.801 & -4.466 & -4.801 & -5.001 & -2.666 & -5.466 \\
\hline \multirow{2}{*}{$\begin{array}{l}\mathrm{E} \\
15 \mu \mathrm{g}\end{array}$} & Sig. & 0.457 & 0.095 & 0.126 & 0.052 & $0.007^{*}$ & $0.026^{*}$ & 0.576 & & $0.011^{*}$ & $0.006^{*}$ & $0.004^{*}$ & 0.193 & $0.001^{*}$ \\
\hline & M.D. ** & -1.066 & -2.401 & 2.201 & -2.801 & -3.866 & -3.201 & 0.801 & & -3.666 & 4.001 & -4.201 & -1.866 & -4.666 \\
\hline \multirow{2}{*}{$\begin{array}{l}\text { R } \\
40 \mu \mathrm{g}\end{array}$} & Sig. & 0.071 & 0.377 & $0.001^{*}$ & 0.545 & 0.889 & 0.744 & $0.002 *$ & $0.011^{*}$ & & 0.816 & 0.710 & 0.211 & 0.485 \\
\hline & M.D. ** & 2.601 & 1.266 & 5.866 & 0.866 & -0.201 & 0.466 & 4.466 & 3.666 & & -0.333 & -0.533 & 1.801 & -1.001 \\
\hline \multirow{2}{*}{$\begin{array}{l}P \\
10 \mu g \\
\end{array}$} & Sig. & $0.042^{*}$ & 0.264 & $0.001^{*}$ & 0.402 & 0.926 & 0.576 & $0.001^{*}$ & $0.006^{*}$ & 0.816 & & 0.889 & 0.137 & 0.642 \\
\hline & M.D. ${ }^{* *}$ & 2.933 & 1.601 & 6.201 & 1.201 & 0.133 & 0.801 & 4.801 & 4.001 & 0.333 & & -0.201 & 2.133 & -0.666 \\
\hline \multirow{2}{*}{$\begin{array}{l}0 \mathrm{~A} \\
2 \mu \mathrm{g} \\
\end{array}$} & Sig. & $0.031^{*}$ & 0.210 & $0.001^{*}$ & 0.329 & 0.816 & 0.485 & $0.001^{*}$ & $0.004^{*}$ & 0.710 & 0.889 & & 0.104 & 0.744 \\
\hline & M.D. ** & 3.133 & 1.801 & 6.401 & 1.401 & 0.333 & 1.001 & 5.001 & 4.201 & 0.533 & 0.201 & & 2.333 & -0.466 \\
\hline \multirow{2}{*}{$\begin{array}{l}\text { B } \\
10 \mu \mathrm{g}\end{array}$} & Sig. & 0.576 & 0.710 & $0.005^{*}$ & 0.515 & 0.163 & 0.352 & 0.064 & 0.193 & 0.211 & 0.137 & 0.104 & & 0.052 \\
\hline & M.D. ** & 0.801 & -0.533 & 4.066 & -0.933 & -2.001 & -1.333 & 2.666 & 1.866 & -1.801 & -2.133 & -2.333 & & -2.801 \\
\hline \multirow{2}{*}{$\begin{array}{l}\mathrm{CC} \\
5 \mu \mathrm{g}\end{array}$} & Sig. & $0.013^{*}$ & 0.115 & $0.001^{*}$ & 0.193 & 0.576 & 0.306 & $0.001^{*}$ & $0.001^{*}$ & 0.485 & 0.642 & 0.744 & 0.052 & \\
\hline & M.D. ${ }^{* *}$ & 3.601 & 2.266 & 6.866 & 1.866 & 0.801 & 1.466 & 5.466 & 4.666 & 1.001 & 0.666 & 0.466 & 2.801 & \\
\hline
\end{tabular}

*The mean difference is significant at the $\mathbf{P}$-Value $=0.05$ level.

**Mean difference.

This table explained the significantly differences between different types of antibiotics when study their activities against Ps. aeruginosa by statistical analysis depended on LSD system. Also showed presences of significant differences between these antibiotics, and this explained through the star that found at p-valve equal or less than 0.05 level However, not all antibiotics have significant differences when comparison with each other, some of them have no significant differences, and this showed through the star that not found at $\mathrm{p}$ valve at 0.05 level.The comparisons involved two types, first the $\mathrm{X}$ side (antibiotics) comparison with $\mathrm{Y}$ side (antibiotics) and the second involved comparison the Y side (antibiotics) with X side (antibiotics).

Figure:2. Show the modes of action of different types of antibiotics against Ps. Aeruginosa

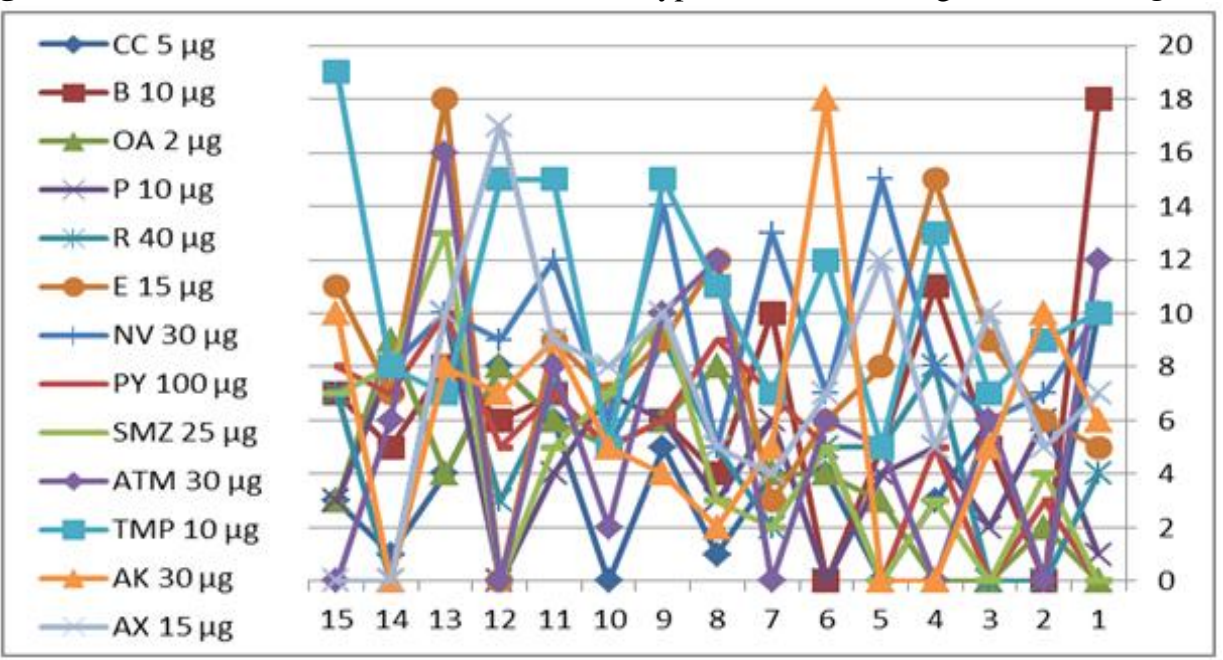

This figure explained the antibiotics types have different modes of actions, this mean each antibiotic showed inhibition zone with different size against same bacteria with several replicates (15 replicates). 
Figure:3. Show the percent of action of different types of antibiotics against Ps. aeruginosa

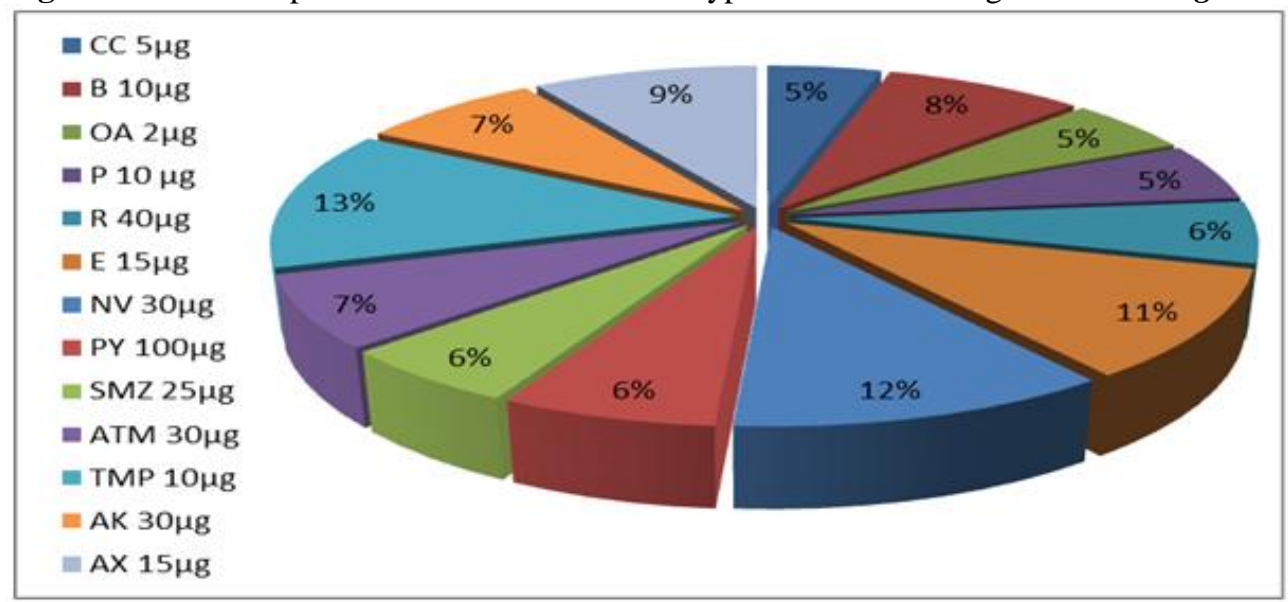

This figure showed the action percentages of different antibiotic types; also explained the TMP 10 antibiotics has high percent equal to $13 \%$ while the CC 5 antibiotic, P 10 antibiotic and OA 2 antibiotic all have low percent equal to 5\% from all antibiotics activities against Ps. Aeruginoa

Table:5. Show the replicates of inhibition zones formed by different types of antibiotics against E.coli

\begin{tabular}{|c|c|c|c|c|c|c|c|c|c|c|c|c|c|}
\hline \multicolumn{14}{|c|}{ Replicates of Inhibition zones measured by $(\mathrm{mm})$} \\
\hline Replicates/* & AX 15 & AK 30 & TMP 10 & ATM 30 & SMZ 25 & PY 100 & NV 30 & E 15 & R 40 & P 10 & $\overline{\mathrm{OA} 2}$ & B 10 & $\mathrm{CC5}$ \\
\hline & 쏘 & 쓰 & 쏘 & 쏘 & $\mu \mathrm{g}$ & 쓰 & $\mu \mathrm{g}$ & 쓰 & 쏘 & 쏘 & 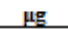 & 쏘 & $\mu \mathrm{g}$ \\
\hline 1 & 7 & 10 & 12 & 9 & 7 & 1 & 0 & 1 & $\mathbf{5}$ & 0 & 2 & 0 & 6 \\
\hline 2 & 10 & 9 & 7 & 8 & 9 & 2 & $\mathbf{5}$ & 1 & 0 & 5 & 5 & 2 & 10 \\
\hline 3 & 8 & 15 & 13 & 7 & 6 & 1 & 3 & 5 & 10 & 10 & 0 & 6 & 0 \\
\hline 4 & 0 & 10 & 0 & 6 & 5 & 3 & 9 & 0 & 0 & 1 & 9 & 0 & 2 \\
\hline 5 & 8 & 11 & 11 & 10 & 12 & 5 & 10 & 1 & 0 & 0 & 5 & 0 & 3 \\
\hline 6 & 12 & 9 & 10 & 9 & 8 & 5 & 5 & $\frac{2}{4}$ & 3 & 4 & 4 & 4 & 4 \\
\hline 7 & 9 & 19 & 8 & 7 & 11 & 4 & 5 & 0 & 6 & 6 & 11 & 3 & 0 \\
\hline 8 & 0 & 7 & 10 & 6 & 6 & 5 & 6 & 8 & 0 & 0 & 0 & 5 & 8 \\
\hline 9 & 13 & 10 & 6 & 9 & 5 & 7 & 7 & 12 & 4 & 5 & 9 & 2 & 2 \\
\hline 10 & 7 & 8 & 12 & 4 & 10 & 5 & 5 & 6 & 2 & 3 & 6 & 6 & 8 \\
\hline 11 & 9 & 12 & 11 & 11 & 8 & $\frac{2}{7}$ & 7 & $\frac{0}{6}$ & 6 & $\frac{2}{7}$ & 0 & $\frac{0}{6}$ & $\frac{0}{6}$ \\
\hline 12 & 10 & 10 & 16 & 13 & 12 & 0 & 4 & 0 & 0 & 10 & 7 & 6 & 0 \\
\hline 13 & 8 & 17 & 15 & 0 & 6 & 0 & 12 & 12 & 2 & 0 & 10 & 0 & 12 \\
\hline 14 & 10 & 14 & 10 & 16 & 8 & 14 & 9 & 12 & 4 & 0 & 0 & 10 & 8 \\
\hline 15 & 11 & 17 & 14 & 22 & 0 & 14 & 7 & 0 & 8 & 10 & 12 & 8 & 16 \\
\hline Mean & 8.134 & 11.867 & 10.333 & 9.134 & 7.534 & 4.867 & 6.267 & 4.534 & 3.334 & 4.067 & 5.334 & 3.867 & 5.667 \\
\hline Median & 9.000 & 11.000 & 11.000 & 9.000 & 8.000 & 5.000 & 6.000 & 4.000 & 3.000 & 4.000 & 5.000 & 4.000 & 6.000 \\
\hline Std. Deviation & 3.719 & 3.661 & 3.995 & 5.123 & 3.137 & 4.356 & 2.988 & 4.657 & 3.199 & 3.899 & 4.271 & 3.182 & 4.746 \\
\hline $\begin{array}{c}\text { Std. Error of } \\
\text { Mean }\end{array}$ & 0.961 & 0.955 & 1.032 & 1.331 & 0.809 & 1.125 & 0.771 & 1.203 & 0.827 & 1.007 & 1.103 & 0.822 & 1.226 \\
\hline
\end{tabular}

This table explained the Mean, Median, Std. deviation and Std. error for replicates of inhibition zones that measured by $(\mathrm{mm})$ for each antibiotics against $E$. coli bacteria. And showed the (AK 30) antibiotic has high activity $(11.867 \mathrm{~mm})$ while the (B 10) antibiotic has low activity $(3.867 \mathrm{~mm})$ against $E$. coli bacteria by mean measured.

\begin{tabular}{|l|l|l|l|}
\hline${ }^{*}$ AX 15 & Amoxicillin15 $\mu \mathrm{g}$ & ${ }^{*}$ E 15 & Erythromycin15 $\mu \mathrm{g}$ \\
\hline${ }^{*}$ AK 30 & Amikacin30 $\mu \mathrm{g}$ & ${ }^{*}$ R 40 & Rifaximin40 $\mu \mathrm{g}$ \\
\hline${ }^{*}$ TMP 10 & Trimethoprim10 $\mu \mathrm{g}$ & ${ }^{*}$ P 10 & Penicillin-G10 $\mu \mathrm{g}$ \\
\hline${ }^{*}$ ATM 30 & Aztreonam30 $\mu \mathrm{g}$ & ${ }^{*}$ OA 2 & Oxolinic acid2 $\mu \mathrm{g}$ \\
\hline${ }^{*}$ SMZ 25 & Sulfamethoxazole25 $\mu \mathrm{g}$ & ${ }^{*}$ B 10 & Bacitracin10 $\mu \mathrm{g}$ \\
\hline *PY 100 & Carbnicillin100 $\mu \mathrm{g}$ & ${ }^{*}$ CC 5 & Clindamycin5 $\mu \mathrm{g}$ \\
\hline *NV 30 & Novobiocin30 $\mu \mathrm{g}$ \\
\hline
\end{tabular}


Table:6. Show the significantly study according to LSD system for different types of antibiotics against $E$. coli

\begin{tabular}{|c|c|c|c|c|c|c|c|c|c|c|c|c|c|c|}
\hline \multicolumn{15}{|c|}{ Significantly study ac } \\
\hline $\begin{array}{l}\text { Y side } \\
\text { (antibio }\end{array}$ & s) $X$ side & $\begin{array}{l}\mathrm{AX15} \\
\mu \mathrm{g}\end{array}$ & $\begin{array}{l}\text { AK30 } \\
\mu \mathrm{g}\end{array}$ & $\begin{array}{l}\text { TMP10 } \\
\mu \mathrm{g}\end{array}$ & $\begin{array}{l}\text { ATM30 } \\
\mu \mathrm{g}\end{array}$ & $\begin{array}{l}\text { SMZ25 } \\
\mu \mathrm{g}\end{array}$ & $\begin{array}{l}\text { PY100 } \\
\text { sg }\end{array}$ & $\begin{array}{l}\text { NV30 } \\
\mu \mathrm{g}\end{array}$ & $\begin{array}{l}\text { E } 15 \\
\mu \mathrm{g}\end{array}$ & $\begin{array}{l}\text { R 40 } \\
\mu g\end{array}$ & $\begin{array}{ll}\begin{array}{l}\text { P } \\
\mu \mathrm{g}\end{array} & 10\end{array}$ & $\begin{array}{l}\text { OA2 } 2 \\
\mu \mathrm{g}\end{array}$ & $\begin{array}{l}\text { B 10 } \\
\mu \mathrm{g}\end{array}$ & $\begin{array}{l}\mathrm{CC} 5 \\
\mu \mathrm{g}\end{array}$ \\
\hline \multicolumn{2}{|c|}{\begin{tabular}{l|l} 
(antibiotics) \\
$\mathbf{A X}$ & Sig.
\end{tabular}} & & $0.011^{*}$ & 0.131 & 1.001 & 0.680 & $0.026^{*}$ & 0.200 & $0.014^{*}$ & $0.001^{*}$ & $0.006^{*}$ & 0.055 & $0.004^{*}$ & -0.091 \\
\hline \multirow{3}{*}{$\begin{array}{l}15 \mu \mathrm{g} \\
\mathrm{AK} \\
30 \mu \mathrm{g}\end{array}$} & M.D. ${ }^{* *}$ & & 3.733 & 2.201 & 0.492 & -0.601 & -3.266 & -1.866 & -3.601 & -4.801 & -4.066 & -2.801 & -4.266 & 2.466 \\
\hline & Sig. & $0.011^{*}$ & & 0.292 & 0.061 & $0.003^{*}$ & $0.011^{*}$ & $0.001^{*}$ & $0.001^{*}$ & $0.001^{*}$ & $0.001^{*}$ & $0.001^{*}$ & $0.001^{*}$ & $0.001^{*}$ \\
\hline & M.D. ${ }^{* *}$ & -3.733 & & -1.533 & -2.733 & -4.333 & -7.00 & -5.601 & -7.333 & -8.533 & -7.801 & -6.533 & -8.001 & -6.201 \\
\hline \multirow{2}{*}{$\begin{array}{l}\text { TMP } \\
10 \mu \mathrm{g}\end{array}$} & Sig. & 0.131 & 0.29 & & 0.410 & 0.055 & $0.011^{*}$ & $0.006^{*}$ & $0.001^{*}$ & $0.001^{*}$ & $0.001^{*}$ & $0.001^{*}$ & $0.001^{*}$ & $0.002^{*}$ \\
\hline & M.D. ${ }^{* *}$ & -2.201 & 1.533 & & -1.201 & -2.801 & -5.466 & -4.066 & -5.801 & -7.001 & -6.266 & -5.001 & -6.466 & -4.666 \\
\hline \multirow{2}{*}{$\begin{array}{l}\text { ATM } \\
30 \mu \mathrm{g}\end{array}$} & Sig. & 0.492 & 0.06 & 0.410 & & 0.272 & $0.004^{*}$ & $0.051^{*}$ & $0.002^{*}$ & $0.001^{*}$ & $0.001^{*}$ & $0.011^{*}$ & $0.001^{*}$ & $0.018^{*}$ \\
\hline & M.D. ${ }^{* *}$ & -1.001 & 2.733 & 1.201 & & -1.601 & -4.266 & -2.866 & -4.601 & -5.801 & -5.066 & -3.801 & -5.266 & -3.466 \\
\hline \multirow{2}{*}{$\begin{array}{l}\text { SMZ } \\
25 \mu \mathrm{g}\end{array}$} & Sig. & 0.681 & $0.003^{*}$ & 0.055 & 0.272 & & 0.068 & 0.384 & $0.014^{*}$ & $0.004^{*}$ & 0.018 & 0.131 & $0.012^{*}$ & 0.200 \\
\hline & M.D. ${ }^{* *}$ & 0.601 & 4.33 & 2.801 & 1.601 & & -2.666 & -1.266 & -3.001 & -4.201 & -3.466 & -2.201 & -3.666 & -1.866 \\
\hline \multirow{2}{*}{$\begin{array}{l}\text { PY } \\
100 \mu \mathrm{g}\end{array}$} & Sig. & $0.026^{*}$ & $0.001^{*}$ & $0.001^{*}$ & $0.004^{*}$ & 0.068 & & 0.336 & 0.819 & 0.292 & 0.582 & 0.748 & 0.492 & 0.582 \\
\hline & M.D. $* *$ & 3.601 & 7.001 & 5.466 & 4.266 & 2.666 & & 1.401 & -0.333 & -1.533 & -0.801 & -0.466 & -1.001 & 1.801 \\
\hline \multirow{2}{*}{$\begin{array}{l}\text { NV } \\
30 \mu \mathrm{g}\end{array}$} & Sig. & 0.201 & $0.001^{*}$ & $0.006^{*}$ & $0.051^{*}$ & 0.384 & 0.336 & & 0.234 & $0.045^{*}$ & 0.131 & 0.521 & 0.101 & 0.680 \\
\hline & M.D. ${ }^{* *}$ & 1.866 & 5.601 & 4.066 & 2.866 & 1.266 & -1.401 & & -1.733 & -2.933 & -2.201 & -0.933 & -2.401 & -0.601 \\
\hline \multirow{2}{*}{$\begin{array}{l}\mathrm{E} \\
15 \mu \mathrm{g}\end{array}$} & Sig. & $0.014^{*}$ & $0.001^{*}$ & $0.001^{*}$ & $0.002 *$ & $0.014^{*}$ & 0.819 & 0.234 & & 0.410 & 0.748 & 0.582 & 0.647 & 0.436 \\
\hline & M.D. $* *$ & 3.601 & 7.333 & 5.801 & 4.601 & 3.001 & 0.333 & -1.733 & & -1.201 & -0.466 & 0.801 & 0.666 & -1.133 \\
\hline \multirow{3}{*}{$\begin{array}{l}\mathrm{R} \\
40 \mu \mathrm{g}\end{array}$} & Sig. & $0.001^{*}$ & $0.001^{*}$ & $0.001^{*}$ & $0.001^{*}$ & $0.004^{*}$ & 0.292 & $0.045^{*}$ & 0.410 & & 0.614 & 0.170 & 0.714 & 0.110 \\
\hline & M.D. ${ }^{* *}$ & 4.801 & 8.533 & 8.533 & 5.801 & 4.201 & 1.533 & 2.933 & 1.201 & & -0.733 & 2.001 & -0.533 & 2.333 \\
\hline & Sig. & $0.006^{\star}$ & $0.001^{*}$ & $0.001^{*}$ & $0.001^{*}$ & 0.018 & 0.582 & 0.131 & 0.748 & 0.614 & & 0.384 & 0.891 & 0.272 \\
\hline $\begin{array}{l}\mathrm{P} \\
10 \mu \mathrm{g}\end{array}$ & M.D. ** & 4.066 & 7.801 & 6.266 & 5.066 & $3.466^{*}$ & 0.801 & 2.201 & 0.466 & -0.733 & & 1.266 & -0.201 & 1.601 \\
\hline \multirow{2}{*}{$\begin{array}{l}\mathrm{OA} \\
2 \mu \mathrm{g} \\
\end{array}$} & Sig. & 0.055 & $0.001^{*}$ & $0.001^{*}$ & $0.011^{*}$ & 0.131 & 0.748 & 0.521 & 0.582 & 0.170 & 0.384 & & 0.314 & 0.819 \\
\hline & M.D. $* *$ & 2.801 & 6.533 & 5.00 & 3.801 & 2.201 & -0.466 & 0.933 & -0.801 & -2.001 & -1.266 & & -1.466 & 0.333 \\
\hline \multirow{2}{*}{$\begin{array}{l}\mathrm{B} \\
10 \mu \mathrm{g}\end{array}$} & Sig. & $0.004^{*}$ & $0.001^{*}$ & $0.001^{*}$ & $0.001^{*}$ & $0.012^{*}$ & 0.492 & 0.101 & 0.647 & 0.714 & 0.891 & 0.314 & & 0.217 \\
\hline & M.D. $* *$ & 4.266 & 8.001 & 6.466 & 5.266 & 3.666 & 1.001 & 2.401 & 0.666 & -0.533 & 0.201 & 1.466 & & 1.801 \\
\hline \multirow{2}{*}{$\begin{array}{l}\mathrm{CC} \\
5 \mu \mathrm{g}\end{array}$} & Sig. & 0.091 & $0.001^{*}$ & $0.002 *$ & $0.018^{*}$ & 0.200 & 0.582 & 0.680 & 0.436 & 0.110 & 0.272 & 0.819 & 0.217 & \\
\hline & M.D. ** & 2.466 & 6.201 & 4.666 & 3.466 & 1.866 & -1.801 & 0.601 & -1.133 & -2.333 & -1.601 & -0.333 & -1.801 & \\
\hline
\end{tabular}

*The mean difference is significant at the $\mathbf{P}-$ Value $=0.05$ level.

**Mean difference.

This table explained the significantly differences between different types of antibiotics when study their activities against $E$. coli by statistical analysis depended on LSD system. Also showed presences of significant differences between these antibiotics, and this explained through the star that found at p-valve equal or less than 0.05 level.However, not all antibiotics have significant differences when comparison with each other, some of them have no significant differences, and this showed through the star that not found at $\mathrm{p}$-valve at 0.05 level. The comparisons involved two types, first the $\mathrm{X}$ side (antibiotics) comparison with $\mathrm{Y}$ side (antibiotics) and the second involved comparison the Y side (antibiotics) with X side (antibiotics).

Figure:4. Show the modes of action of different types of antibiotics against E. coli

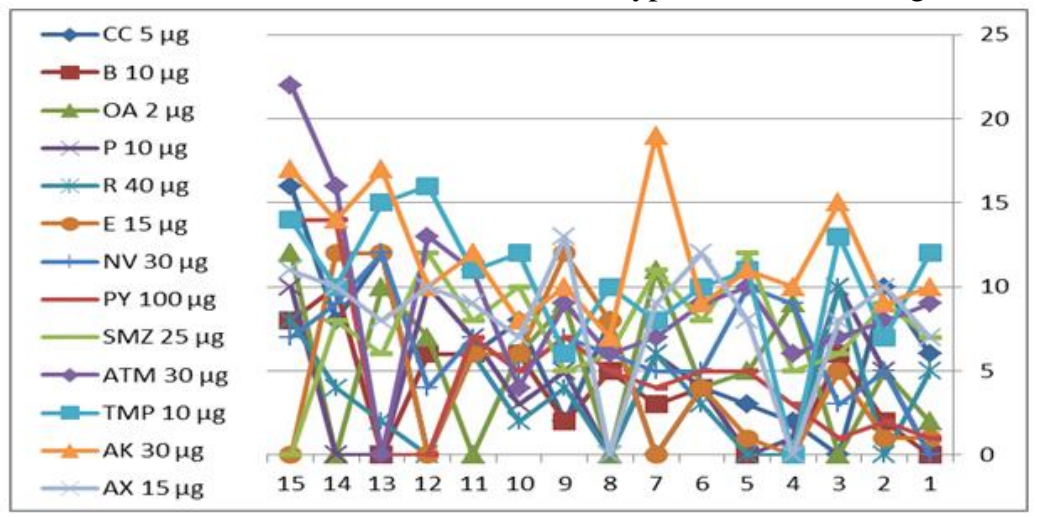

This figure explained the antibiotics types have different modes of actions, this mean each antibiotic showed inhibition zone with different size against same bacteria with several replicates (15 replicates).

Figure:5. Show the percent of action of different types of antibiotics against E. coli

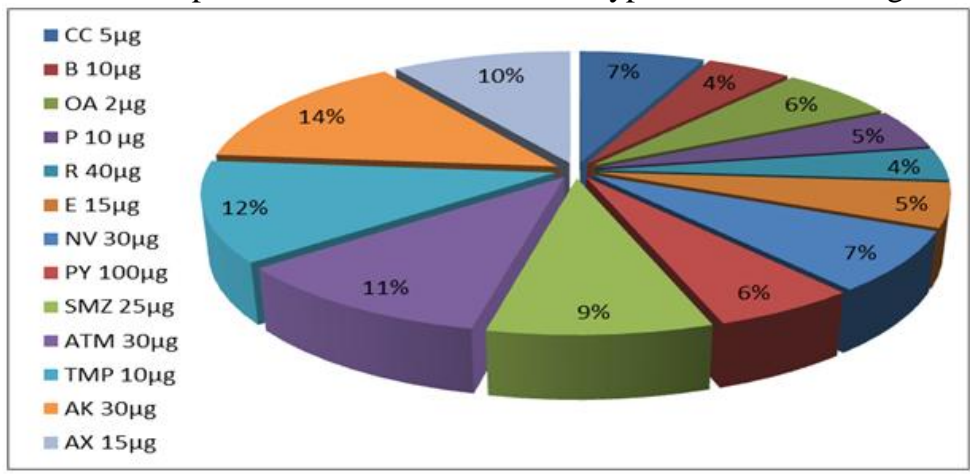


Isolation, Identification and Antibiotic Susceptibility of pathogenic Bacteria Isolated from Clinical..

This figure showed the action percentages of different antibiotic types; also explained the AK 30 antibiotics has high percent equal to $14 \%$ while the B10 antibiotic and R 40 antibiotic both have low percent equal to $4 \%$ from all antibiotics activities against $E$. coli.

Table:7. Show the replicates of inhibition zones formed by different types of antibiotics against Enterobacter cloacae

\begin{tabular}{|c|c|c|c|c|c|c|c|c|c|c|c|c|c|}
\hline \multicolumn{14}{|c|}{ Replicates of Inhibition zones measured by $(\mathrm{mm})$} \\
\hline Replicates/* & $\underset{\mu \mathrm{g}}{\mathrm{AX} 15}$ & $\begin{array}{c}\text { AK 30 } \\
\mu \mathrm{g}\end{array}$ & $\begin{array}{c}\text { TMP 10 } \\
\mu \mathrm{g}\end{array}$ & ATM $30 \mu \mathrm{g}$ & $\begin{array}{c}\text { SMZ 25 } \\
\mu \mathrm{g}\end{array}$ & $\begin{array}{c}\text { PY } 100 \\
\mu \mathrm{g}\end{array}$ & $\begin{array}{c}\text { NV 30 } \\
\mu \mathrm{g}\end{array}$ & $\begin{array}{c}E 15 \\
\mu \mathrm{g}\end{array}$ & $\begin{array}{c}\text { R 40 } \\
\mu \mathrm{g}\end{array}$ & $\begin{array}{c}P 10 \\
\mu \mathrm{g}\end{array}$ & $\begin{array}{c}\text { OA2 } \\
\mu \mathrm{g}\end{array}$ & $\begin{array}{c}\text { B 10 } \\
\text { Hg }\end{array}$ & $\begin{array}{l}\mathrm{CC5} 5 \\
\mu \mathrm{g}\end{array}$ \\
\hline 1 & 6 & 6 & 13 & 15 & 5 & 0 & 5 & 10 & 9 & 6 & 5 & 0 & 0 \\
\hline 2 & 7 & 3 & 7 & 13 & 7 & 7 & 9 & 8 & 6 & 5 & 6 & 5 & 7 \\
\hline 3 & 3 & 0 & 9 & 16 & 10 & 0 & 4 & 9 & 4 & 10 & 13 & 0 & 3 \\
\hline 4 & 9 & 8 & 12 & 20 & 9 & 0 & 7 & 7 & 7 & 4 & 3 & 3 & 0 \\
\hline 5 & 8 & 7 & 11 & 18 & 4 & 5 & 3 & 6 & 5 & 6 & 4 & 9 & 3 \\
\hline 6 & 9 & 7 & 9 & 20 & 0 & 6 & 8 & 10 & 10 & 7 & 8 & 5 & 6 \\
\hline 7 & 14 & 14 & 10 & 15 & 10 & 5 & 16 & 15 & 15 & 0 & 1 & 5 & 3 \\
\hline 8 & 0 & 0 & 8 & 25 & 4 & 1 & 6 & 8 & 18 & 4 & 6 & 3 & 9 \\
\hline 9 & 15 & 8 & 10 & 17 & 5 & 11 & 0 & 14 & 13 & 12 & 15 & 2 & 2 \\
\hline 10 & 8 & 6 & 8 & 23 & 9 & 7 & 10 & 7 & 6 & 9 & 10 & 0 & 10 \\
\hline 11 & 12 & 9 & 6 & 22 & 5 & 8 & 10 & 12 & 8 & 9 & 5 & 7 & 8 \\
\hline 12 & 5 & 7 & 9 & 30 & 7 & 3 & 15 & 10 & 12 & 2 & 15 & 11 & 0 \\
\hline 13 & 10 & 11 & 0 & 14 & 6 & 16 & 6 & 6 & 18 & 8 & 9 & 1 & 16 \\
\hline 14 & 19 & 8 & 16 & 23 & 11 & 0 & 13 & 18 & 5 & 5 & 4 & 2 & 11 \\
\hline 15 & 15 & 10 & 5 & 21 & 3 & 5 & 7 & 14 & 6 & 4 & 8 & 11 & 5 \\
\hline Mean & 9.333 & 6.934 & 8.867 & 19.466 & 6.333 & 4.934 & 7.933 & 10.267 & 9.466 & 6.067 & 7.466 & 4.253 & 5.534 \\
\hline Median & 9.000 & 7.000 & 9.000 & 20.000 & 6.000 & 5.000 & 7.000 & 10.000 & 8.000 & 6.000 & 6.000 & 3.000 & 5.000 \\
\hline Std. Deviation & 5.023 & 3.751 & 3.701 & 4.688 & 3.062 & 4.589 & 4.399 & 3.614 & 4.703 & 3.788 & 4.274 & 3.788 & 4.657 \\
\hline $\begin{array}{l}\text { Std. Error of } \\
\text { Mean }\end{array}$ & 1.297 & 0.968 & 0.955 & 1.211 & 0.7908 & 1.185 & 1.135 & 0.933 & 1.214 & 0.819 & 1.104 & 0.978 & 1.203 \\
\hline
\end{tabular}

This table explained the Mean, Median, Std. deviation and Std. error for replicates of inhibition zones that measured by $(\mathrm{mm})$ for each antibiotics against Enterobacter cloacae bacteria. And showed the (ATM 30) antibiotic has high activity $(19.466 \mathrm{~mm})$ while the (PY 100) antibiotic has low activity (4.934 mm) against Enterobacter cloacae bacteria by mean measured.

\begin{tabular}{|c|c|c|c|}
\hline *AX 15 & Amoxicillin15 $\mu \mathrm{g}$ & ${ }^{*}$ E 15 & Erythromycin15 $\mu \mathrm{g}$ \\
\hline *AK 30 & Amikacin30 $\mu \mathrm{g}$ & ${ }^{*} \mathbf{R} 40$ & Rifaximin40 $\mu \mathrm{g}$ \\
\hline${ }^{*}$ TMP 10 & Trimethoprim10 $\mu \mathrm{g}$ & ${ }^{*} \mathbf{P} 10$ & Penicillin-G10 $\mu \mathrm{g}$ \\
\hline *ATM 30 & Aztreonam30 $\mu \mathrm{g}$ & *OA 2 & Oxolinic acid2 $\mu \mathrm{g}$ \\
\hline *SMZ 25 & Sulfamethoxazole $25 \mu \mathrm{g}$ & *B 10 & Bacitracin10 $\mu \mathrm{g}$ \\
\hline *PY 100 & Carbnicillin100 $\mu \mathrm{g}$ & $* \mathrm{CC} 5$ & Clindamycin5 $\mu \mathrm{g}$ \\
\hline *NV 30 & \multicolumn{3}{|l|}{ Novobiocin30 $\mu \mathrm{g}$} \\
\hline
\end{tabular}

Table:8. Show the significantly study according to LSD system for different types of antibiotics against Enterobacter cloacae

\begin{tabular}{|c|c|c|c|c|c|c|c|c|c|c|c|c|c|c|}
\hline \multicolumn{15}{|c|}{ Significantly study according to LSD at P-Value $=0.05$} \\
\hline \multicolumn{2}{|c|}{$\begin{array}{c}\text { Y side / X side } \\
\text { (antibiotics) }\end{array}$} & $\underset{\mu \mathrm{g}}{\mathrm{AX15}}$ & $\begin{array}{c}\mathrm{AK3} 30 \\
\mu \mathrm{g}\end{array}$ & $\begin{array}{c}\text { TMP10 } \\
\mu \mathrm{g}\end{array}$ & $\begin{array}{c}\text { ATM30 } \\
\mu \mathrm{g}\end{array}$ & $\begin{array}{c}\text { SMZ2 } \\
5 \mu \mathrm{g}\end{array}$ & $\begin{array}{c}\text { PY100 } \\
\mu \mathrm{g}\end{array}$ & $\begin{array}{c}\text { NV30 } \\
\mu \mathrm{g}\end{array}$ & $\begin{array}{c}\text { E } 15 \\
\mu \mathrm{g}\end{array}$ & $\begin{array}{c}\mathrm{R} 40 \\
\mu \mathrm{g}\end{array}$ & $\begin{array}{c}P 10 \\
\mu \mathrm{g}\end{array}$ & $\begin{array}{c}\mathrm{OA2}^{2} \\
\mu \mathrm{g}\end{array}$ & $\begin{array}{c}\text { B } 10 \\
\mu \mathrm{g}\end{array}$ & $\mathrm{CC5}$ \\
\hline \multirow{2}{*}{$\begin{array}{c}\mathrm{AX} \\
15 \mu \mathrm{g}\end{array}$} & Sig. & & 0.115 & 0.759 & $0.001^{*}$ & $0.049^{*}$ & $0.004^{*}$ & 0.357 & 0.539 & 0.930 & $0.033^{*}$ & 0.220 & $0.001^{*}$ & $0.013^{*}$ \\
\hline & M.D. ** & & -2.401 & -0.466 & -10.133 & -3.001 & -4.401 & -1.401 & 0.933 & 0.133 & -3.266 & -1.866 & -5.066 & -3.801 \\
\hline \multirow{2}{*}{$\begin{array}{c}\text { AK } \\
30 \mu \mathrm{g}\end{array}$} & Sig. & 0.115 & & 0.204 & $0.001^{*}$ & 0.693 & 0.189 & 0.357 & $0.029^{*}$ & 0.097 & 0.568 & 0.726 & 0.080 & 0.357 \\
\hline & M.D. ** & 2.401 & & 1.933 & -12.533 & -0.601 & -2.001 & 1.401 & 3.333 & 2.533 & -0.866 & 0.533 & -2.666 & -1.401 \\
\hline \multirow{2}{*}{$\begin{array}{l}\text { TMP } \\
10 \mu \mathrm{g}\end{array}$} & Sig. & 0.759 & 0.204 & & $0.001^{*}$ & 0.097 & $0.011^{*}$ & 0.539 & 0.357 & 0.693 & 0.067 & 0.357 & $0.003^{*}$ & $0.029^{*}$ \\
\hline & M.D. $* *$ & 0.466 & -1.933 & & 10.600 & -2.533 & -3.933 & -0.933 & 1.401 & 0.601 & -2.801 & -1.401 & -4.601 & -3.333 \\
\hline \multirow{2}{*}{$\begin{array}{l}\text { ATM } \\
30 \mu \mathrm{g}\end{array}$} & Sig. & $0.001^{*}$ & $0.001^{*}$ & $0.001^{*}$ & & $0.001^{*}$ & $0.001^{*}$ & $0.001^{*}$ & $0.001^{*}$ & $0.001^{*}$ & $0.001^{*}$ & $0.001^{*}$ & $0.001^{*}$ & $0.001^{*}$ \\
\hline & M.D. $* *$ & 10.133 & 12.533 & -10.600 & & 13.133 & 14.533 & 11.533 & -9.201 & $10 \overline{001}$ & $\begin{array}{c}- \\
13.401\end{array}$ & 12.001 & 15.201 & -13.933 \\
\hline \multirow{2}{*}{$\begin{array}{r}\text { SMZ } \\
25 \mu \mathrm{g}\end{array}$} & Sig. & $0.049 *$ & 0.693 & 0.097 & $0.001^{*}$ & & 0.357 & 0.293 & $0.011^{*}$ & $0.041^{*}$ & 0.861 & 0.456 & 0.175 & 0.599 \\
\hline & M.D. ** & 3.001 & 0.601 & 2.533 & 13.133 & & -1.401 & 1.601 & 3.933 & 3.133 & 0.266 & 1.133 & -2.066 & -0.801 \\
\hline \multirow{2}{*}{$\begin{array}{c}\text { PY } \\
100 \mu \mathrm{g}\end{array}$} & Sig. & $0.004^{*}$ & 0.189 & $0.011^{*}$ & $0.001^{*}$ & 0.357 & & $0.049 *$ & $0.011^{*}$ & $0.003^{*}$ & 0.456 & 0.097 & 0.661 & 0.693 \\
\hline & M.D. ** & 4.401 & 2.001 & 3.933 & 14.533 & 1.401 & & 3.001 & 5.333 & 4.533 & 1.133 & 2.533 & -0.666 & 0.601 \\
\hline \multirow{2}{*}{$30 \mu \mathrm{g}$} & Sig. & 0.357 & 0.357 & 0.539 & $0.001^{*}$ & 0.293 & $0.049 *$ & & 0.126 & 0.313 & 0.220 & 0.759 & $0.017^{*}$ & 0.115 \\
\hline & M.D. *** & 1.401 & 1.401 & 0.933 & 11.533 & -1.601 & -3.001 & & 2.333 & 1.533 & -1.866 & -0.466 & -3.666 & -2.401 \\
\hline \multirow{3}{*}{$\begin{array}{c}E \\
15 \mu \mathrm{g} \\
\mathrm{R}\end{array}$} & Sig. & 0.539 & $0.029^{*}$ & 0.357 & $0.001^{*}$ & $0.011^{*}$ & $0.011^{*}$ & 0.126 & & 0.599 & $0.006^{*}$ & 0.067 & $0.001^{*}$ & 0.002 \\
\hline & M.D. ** & -0.933 & -3.333 & -1.401 & 9.201 & -3.933 & -5.333 & -2.333 & & -0.801 & -4.201 & -2.801 & -6.001 & -4.733 \\
\hline & Sig. & 0.930 & 0.097 & 0.693 & $0.001^{*}$ & $0.041^{*}$ & $0.003^{*}$ & 0.313 & 0.599 & & $0.026^{*}$ & 0.189 & $0.001^{*}$ & $0.011^{*}$ \\
\hline $40 \mu \mathrm{g}$ & M.D. ${ }^{* *}$ & -0.133 & -2.533 & -0.601 & 10.001 & -3.133 & -4.533 & -1.533 & 0.801 & & -3.401 & -2.001 & -5.201 & -3.933 \\
\hline \multirow{2}{*}{$\begin{array}{c}P \\
10 \mu \mathrm{g}\end{array}$} & Sig. & $0.033^{*}$ & 0.568 & 0.067 & $0.001^{*}$ & 0.861 & 0.456 & 0.220 & $0.006^{*}$ & $0.026^{*}$ & & 0.357 & 0.237 & 0.726 \\
\hline & M.D. ${ }^{* *}$ & 3.266 & 0.866 & 2.801 & 13.401 & 0.266 & -1.133 & 1.866 & 4.201 & 3.401 & & 1.401 & -1.801 & -0.533 \\
\hline \multirow{2}{*}{$\begin{array}{l}\mathrm{OA} \\
2 \mu \mathrm{g}\end{array}$} & Sig. & 0.220 & 0.726 & 0.357 & $0.001^{*}$ & 0.456 & 0.097 & 0.759 & 0.067 & 0.189 & 0.357 & & $0.036^{*}$ & 0.204 \\
\hline & M.D. ** & 1.866 & -0.533 & 1.401 & 12.001 & -1.133 & -2.533 & 0.466 & 2.801 & 2.001 & -1.401 & & -3.201 & -1.933 \\
\hline \multirow{2}{*}{$\begin{array}{c}\mathrm{B} \\
10 \mu \mathrm{g}\end{array}$} & Sig. & $0.001^{*}$ & 0.080 & $0.003^{*}$ & $0.001^{*}$ & 0.175 & 0.661 & $0.017^{*}$ & $0.001^{*}$ & $0.001^{*}$ & 0.237 & $0.036^{*}$ & & 0.405 \\
\hline & M.D. ** & 5.066 & 2.666 & 4.601 & 15.201 & 2.066 & 0.666 & 3.666 & 6.001 & 5.201 & 1.801 & 3.201 & & 1.266 \\
\hline \multirow{2}{*}{$\begin{array}{c}\mathrm{CC} \\
5 \mu \mathrm{g}\end{array}$} & Sig. & $0.013^{*}$ & 0.357 & $0.029^{*}$ & $0.001^{*}$ & 0.599 & 0.693 & 0.115 & 0.002 & $0.011^{*}$ & 0.726 & 0.204 & 0.405 & \\
\hline & M.D. ${ }^{* *}$ & 3.801 & 1.401 & 3.333 & 13.933 & 0.801 & -0.601 & 2.401 & 4.733 & 3.933 & 0.533 & 1.933 & -1.266 & \\
\hline
\end{tabular}

*The mean difference is significant at the $\mathbf{P}$-Value $=\mathbf{0 . 0 5}$ level.

**Mean difference. 
This table explained the significantly differences between different types of antibiotics when study their activities against E. cloacae by statistical analysis depended on LSD system. Also showed presences of significant differences between these antibiotics, and this explained through the star that found at $\mathrm{p}$-valve equal or less than 0.05 level.However, not all antibiotics have significant differences when comparison with each other, some of them have no significant differences, and this showed through the star that not found at $\mathrm{p}$-valve at 0.05 level.The comparisons involved two types, first the $\mathrm{X}$ side (antibiotics) comparison with $\mathrm{Y}$ side (antibiotics) and the second involved comparison the Y side (antibiotics) with $\mathrm{X}$ side (antibiotics).

Figure:6. Show the modes of action of different types of antibiotics against Enterobacter cloacae

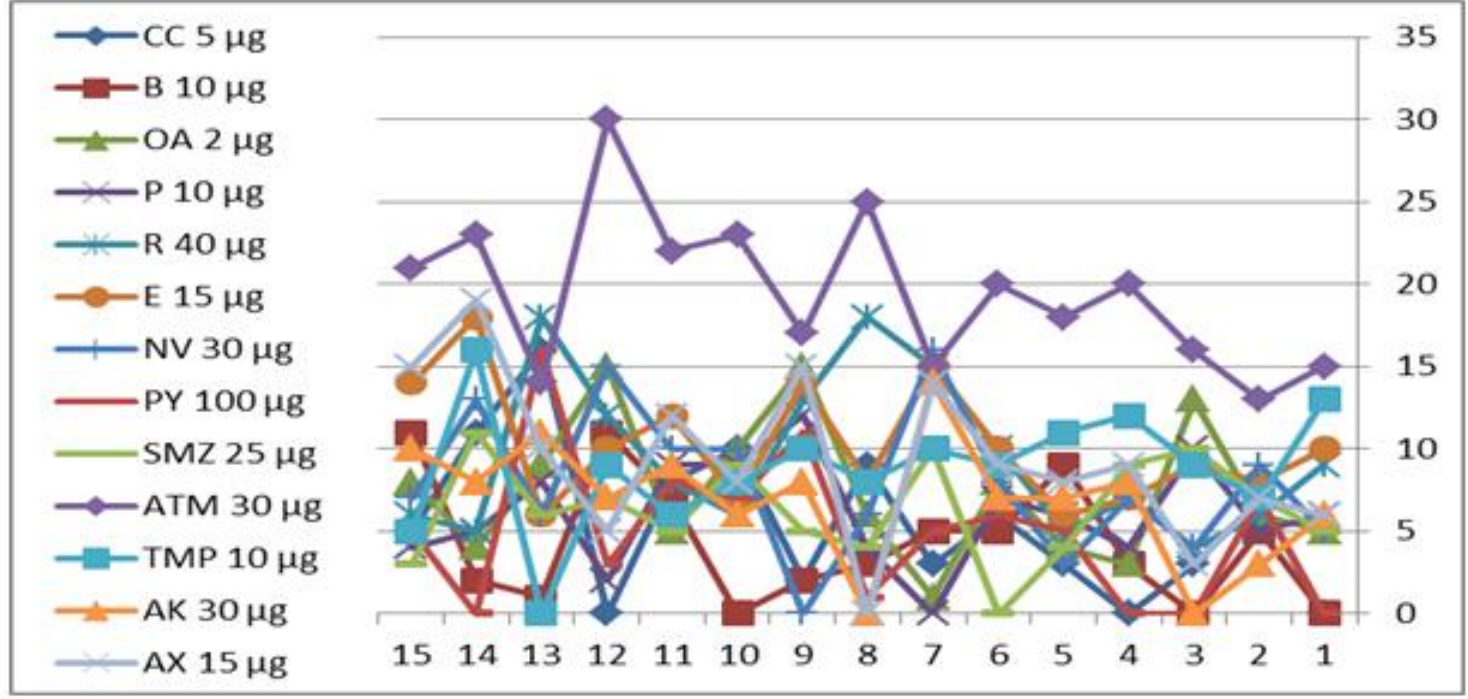

This figure explained the antibiotics types have different modes of actions, this mean each antibiotic showed inhibition zone with different size against same bacteria with several replicates (15 replicates).

Figure:7. Show the percent of action of different types of antibiotics against Enterobacter cloacae

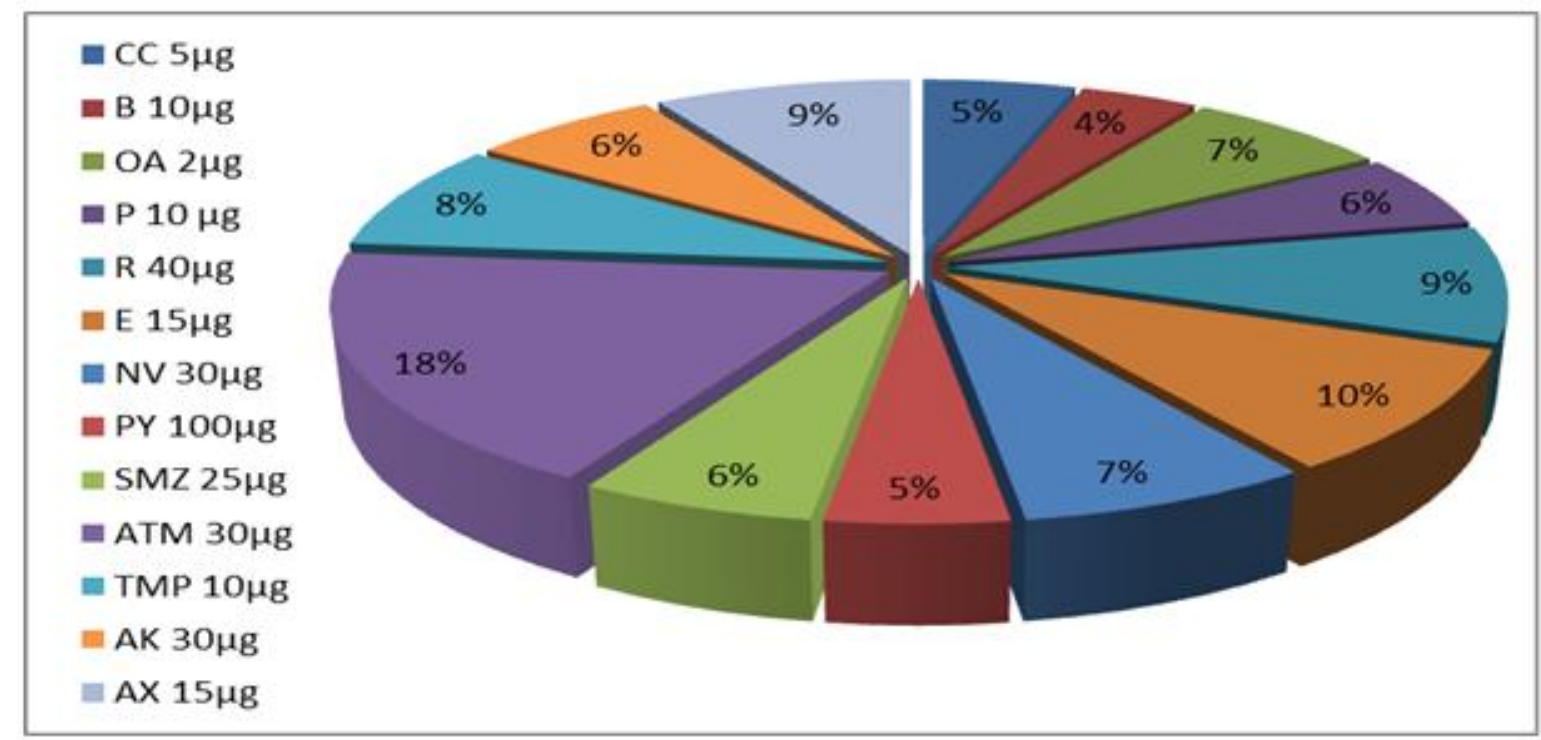

This figure showed the action percentages of different antibiotic types; also explained the ATM 30 antibiotics has high percent equal to $18 \%$ while the B10 antibiotic has low percent equal to $4 \%$ from all antibiotics activities against Enterobacter cloacae. 
Isolation, Identification and Antibiotic Susceptibility of pathogenic Bacteria Isolated from Clinical..

Table:9. Show the replicates of inhibition zones formed by different types of antibiotics against Proteus mirabilis

\begin{tabular}{|c|c|c|c|c|c|c|c|c|c|c|c|c|c|}
\hline \multicolumn{14}{|c|}{ Replicates of Inhibition zones measured by (mm) } \\
\hline Replicates/* & AX 15 & AK 30 & TMP 10 & ATM 30 & SMZ 25 & PY 100 & NV 30 & E 15 & R40 & P10 & OA2 & $\mid$ B 10 & CC5 \\
\hline & $\mu \mathrm{g}$ & $\mu \mathrm{g}$ & $\mu \mathrm{g}$ & $\mu \mathrm{g}$ & $\mu \mathrm{g}$ & $\mu \mathrm{g}$ & $\mu \mathrm{g}$ & $\mu \mathrm{g}$ & $\mu \mathrm{g}$ & $\mu \mathrm{g}$ & $\mu \mathrm{g}$ & $\mu \mathrm{g}$ & $\mu \mathrm{g}$ \\
\hline 1 & 6 & 8 & 0 & 8 & 0 & 6 & 11 & 8 & 12 & 8 & 16 & 11 & 8 \\
\hline$\overline{2}$ & $\bar{~} 0$ & $\bar{~} \overline{5}$ & $\bar{~} 5$ & $\overline{77}$ & 9 & $\overline{12}$ & $\overline{9}$ & $\overline{9}$ & 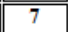 & $\overline{5}$ & 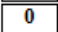 & 6 & $\overline{7}$ \\
\hline 3 & 10 & 6 & 6 & 15 & 13 & 5 & 20 & 7 & 20 & 8 & 12 & 5 & 6 \\
\hline $\bar{~} 4$ & 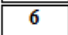 & 8 & $\overline{0}$ & 3 & 12 & 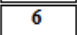 & 14 & 8 & 9 & $\bar{~} \overline{5}$ & 6 & $\overline{0}$ & 4 \\
\hline 5 & $\overline{5}$ & $\overline{c 6}$ & $\overline{88}$ & $\overline{0}$ & 6 & $\overline{5}$ & 19 & 15 & 7 & $\overline{0}$ & $\overline{5}$ & $\overline{5}$ & 10 \\
\hline$\overline{c 6}$ & 7 & 7 & $\bar{~} 6$ & 11 & 8 & 9 & 13 & 11 & 10 & 7 & 7 & 7 & 7 \\
\hline 7 & 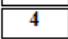 & 8 & 0 & $\overline{10}$ & $\overline{0}$ & $\overline{5}$ & 14 & 9 & 13 & 3 & 4 & $\overline{5}$ & 5 \\
\hline 8 & 10 & 12 & 12 & 12 & 16 & 0 & 6 & 11 & 8 & 7 & 12 & 9 & 8 \\
\hline 9 & 5 & $\overline{14}$ & 5 & $\overline{16}$ & 6 & 10 & $\overline{16}$ & $\overline{16}$ & 9 & $\overline{0}$ & 9 & 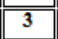 & 9 \\
\hline$\overline{10}$ & 9 & 6 & $\overline{77}$ & 14 & 14 & 7 & 10 & 18 & 14 & $\overline{5}$ & 8 & 0 & 10 \\
\hline 11 & 9 & $\overline{10}$ & 8 & 10 & 10 & 9 & 16 & 11 & $\overline{13}$ & 7 & 11 & $\overline{11}$ & 12 \\
\hline 12 & 16 & 15 & 6 & 0 & 0 & 8 & 7 & 15 & 15 & 0 & $\overline{5}$ & $\bar{~} 5$ & 8 \\
\hline 13 & 2 & 5 & $\overline{10}$ & 7 & 15 & 2 & 9 & 9 & 13 & 8 & 7 & 17 & 13 \\
\hline $1 \overline{14}$ & 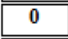 & 20 & 16 & 14 & 6 & 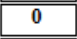 & 12 & 7 & 11 & 6 & 10 & 8 & 6 \\
\hline 15 & 8 & $\begin{array}{ll}0 \\
\end{array}$ & $\overline{0}$ & 16 & 7 & 20 & 15 & 0 & 9 & 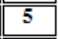 & $\begin{array}{ll}0 \\
\end{array}$ & 14 & 11 \\
\hline Mean & 6.467 & 8.667 & 5.933 & 9.534 & 8.134 & 6.934 & 12.734 & 10.2667 & 11.334 & 4.934 & 7.467 & 7.067 & 8.266 \\
\hline Median & 6.000 & 8.000 & $\overline{60.000}$ & 10.000 & 8.000 & 6.000 & 13.000 & 9.000 & 11.000 & 5.000 & 7.000 & 6.000 & 8.000 \\
\hline $\begin{array}{c}\text { Std. } \\
\text { Deviation }\end{array}$ & 4.189 & 4.909 & 4.683 & 5.343 & 5.344 & 4.994 & 4.132 & 4.479 & 3.499 & 2.915 & 4.422 & 4.758 & \\
\hline $\begin{array}{c}\text { Std. Error of } \\
\text { Mean } \\
\end{array}$ & 1.082 & 1.167 & 1.109 & 1.384 & 1.379 & 1.289 & 1.067 & 1.157 & 0.904 & 0.753 & 1.142 & 1.129 & \\
\hline
\end{tabular}

This table explained the Mean, Median, Std. deviation and Std. error for replicates of inhibition zones that measured by (mm) for each antibiotics against Proteus mirabilis bacteria. And showed the (NV 30) antibiotic has high activity $(12.734 \mathrm{~mm})$ while the (P 10) antibiotic has low activity (4.934 $\mathrm{mm})$ against Proteus mirabilis bacteria by mean measured.

\begin{tabular}{|l|l|l|l|}
\hline${ }^{*}$ AX 15 & Amoxicillin15 $\mu \mathrm{g}$ & ${ }^{*}$ E 15 & Erythromycin15 $\mu \mathrm{g}$ \\
\hline${ }^{\text {AK 30 }}$ & Amikacin30 $\mu \mathrm{g}$ & ${ }^{*}$ R 40 & Rifaximin40 $\mu \mathrm{g}$ \\
\hline${ }^{*}$ TMP 10 & Trimethoprim10 $\mu \mathrm{g}$ & ${ }^{*}$ P 10 & Penicillin-G10 $\mu \mathrm{g}$ \\
\hline${ }^{*}$ ATM 30 & Aztreonam30 $\mu \mathrm{g}$ & ${ }^{*}$ OA 2 & Oxolinic acid2 $\mu \mathrm{g}$ \\
\hline${ }^{*}$ SMZ 25 & Sulfamethoxazole25 $\mu \mathrm{g}$ & ${ }^{*}$ B 10 & Bacitracin10 $\mu \mathrm{g}$ \\
\hline *PY 100 & Carbnicillin100 $\mu \mathrm{g}$ & ${ }^{*}$ CC 5 & Clindamycin5 $\mu \mathrm{g}$ \\
\hline *NV 30 & Novobiocin30 $\mu \mathrm{g}$ & \multicolumn{3}{|l}{} \\
\hline
\end{tabular}

Table:10. Show the significantly study according to LSD system for different types of antibiotics against Proteus mirabilis

\begin{tabular}{|c|c|c|c|c|c|c|c|c|c|c|c|c|c|c|}
\hline \multicolumn{15}{|c|}{ Significantly study according to LSD at P-Value $=0.05$} \\
\hline \multirow{2}{*}{\multicolumn{2}{|c|}{$\begin{array}{c}\text { Y side / X side } \\
\text { (antibiotics) }\end{array}$}} & \multirow{3}{*}{$\begin{array}{c}\mathbf{A X 1 5} \\
\mu \mathrm{g}\end{array}$} & \multirow{3}{*}{$\begin{array}{c}\begin{array}{c}\text { AK30 } \\
\mu \mathrm{g}\end{array} \\
0.173\end{array}$} & \multirow{3}{*}{\begin{tabular}{|c|} 
TMP10 \\
$\mu \mathrm{g}$
\end{tabular}} & \multirow{3}{*}{\begin{tabular}{|c|} 
ATM30 \\
$\mu \mathrm{g}$ \\
0.058
\end{tabular}} & \multirow{3}{*}{\begin{tabular}{|c|} 
SMZ25 \\
$\mu \mathrm{g}$ \\
0.301
\end{tabular}} & \multirow{3}{*}{\begin{tabular}{|c|} 
PY100 \\
$\mu \mathrm{g}$
\end{tabular}} & \multirow{3}{*}{\begin{tabular}{|c|}
$\begin{array}{c}\text { NV30 } \\
\mu \mathrm{g}\end{array}$ \\
$0.001^{\circ}$
\end{tabular}} & \multirow{3}{*}{\begin{tabular}{|c|}
$\begin{array}{c}\text { E15 } \\
\mu \mathrm{g}\end{array}$ \\
$0.019^{\circ}$ \\
\end{tabular}} & \multirow{3}{*}{\begin{tabular}{|c|}
$\begin{array}{c}\text { R 40 } \\
\mu \mathrm{g}\end{array}$ \\
$0.003^{\circ}$
\end{tabular}} & \multirow{3}{*}{\begin{tabular}{|c|}
$\begin{array}{c}\text { P10 } \\
\mu \mathrm{g}\end{array}$ \\
0.341
\end{tabular}} & \multirow{3}{*}{$\begin{array}{c}\begin{array}{c}O A_{2} 2 \\
\mu \mathrm{g}\end{array} \\
0.535\end{array}$} & \multirow{3}{*}{$\begin{array}{c}\begin{array}{c}\text { B10 } \\
\mu \mathrm{g}\end{array} \\
0.709\end{array}$} & \multirow{3}{*}{$\begin{array}{c}\begin{array}{c}\text { CC 5 } \\
\mu \mathrm{g}\end{array} \\
0.264\end{array}$} \\
\hline & & & & & & & & & & & & & & \\
\hline AX & Sig. & & & & & & & & & & & & & \\
\hline $15 \mu \mathrm{g}$ & M.D. ${ }^{* * 8}$ & & 2.200 & -0.533 & 3.066 & 1.666 & 0.466 & 6.266 & 3.801 & 4.866 & -1.533 & 1.001 & 0.600 & 1.801 \\
\hline \multirow{2}{*}{$\begin{array}{c}\mathrm{AK} \\
30 \mu \mathrm{g}\end{array}$} & Sig. & 0.173 & & 0.091 & 0.590 & 0.740 & 0.282 & $0.012^{3}$ & 0.321 & 0.099 & $0.021^{*}$ & 0.456 & 0.321 & 0.803 \\
\hline & M.D. ${ }^{2 *}$ & -2.200 & & -2.733 & 0.866 & -0.533 & -1.733 & 4.066 & 1.601 & 2.666 & -3.733 & -1.201 & -1.601 & 0.401 \\
\hline \multirow{2}{*}{$\begin{array}{l}\text { TMP } \\
10 \mu \mathrm{g}\end{array}$} & Sig. & 0.740 & 0.091 & & $0.026^{x}$ & 0.173 & 0.535 & $0.001^{*}$ & $0.007^{\circ}$ & $0.001^{3}$ & 0.535 & 0.341 & 0.482 & 0.148 \\
\hline & M.D. ${ }^{88}$ & 0.533 & 2.733 & & 3.601 & 2.201 & 1.001 & 6.801 & \begin{tabular}{|c|}
4333 \\
\end{tabular} & \begin{tabular}{|l|l|}
5.401 \\
\end{tabular} & -1.001 & 1.533 & 1.133 & 2.333 \\
\hline \multirow{2}{*}{$\begin{array}{l}\text { ATM } \\
30 \mu \mathrm{g} \\
\end{array}$} & Sig. & 0.058 & 0.590 & $0.026^{\circ}$ & & 0.385 & 0.107 & $0.048^{\circ}$ & 0.649 & 0.264 & $0.004^{2}$ & 0.201 & 0.127 & 0.432 \\
\hline & M.D. ${ }^{88}$ & -3.066 & -0.866 & -3.601 & & -1.401 & -2.601 & 3.201 & 0.733 & 1.801 & -4.601 & -2.066 & -2.466 & -1.266 \\
\hline \multirow{2}{*}{$\begin{array}{l}\mathrm{SMZ} \\
25 \mu \mathrm{g}\end{array}$} & Sig. & 0.301 & 0.740 & 0.173 & 0.385 & & 0.456 & $0.004^{8}$ & 0.186 & $0.048^{\circ}$ & $0.048^{\circ}$ & 0.679 & 0.508 & 0.934 \\
\hline & M.D. ${ }^{* *}$ & -1.666 & 0.533 & -2.201 & 1.401 & & -1.201 & 4.601 & 2.133 & 3.201 & $\mid-3.201$ & -0.666 & -1.066 & 0.133 \\
\hline \multirow{2}{*}{\begin{tabular}{c|}
$\mathrm{PY}$ \\
100 \\
$\mu \mathrm{g}$
\end{tabular}} & Sig. & 0.772 & 0.282 & 0.535 & 0.107 & $\overline{0.456}$ & & $0.001^{*}$ & $0.041^{\circ}$ & $0.007^{2}$ & 0.215 & 0.741 & 0.934 & 0.408 \\
\hline & M.D. ${ }^{8 *}$ & -0.466 & 1.733 & -1.001 & 2.601 & 1.201 & & 5.801 & 3.333 & 4.401 & -2.001 & 0.533 & 0.133 & 1.333 \\
\hline \multirow{2}{*}{$\begin{array}{c}N V \\
30 \mu \mathrm{g} \\
\end{array}$} & Sig. & $0.001^{8}$ & $0.012^{2}$ & $0.001^{8}$ & $0.048^{2}$ & $0.004^{2}$ & $0.001^{2}$ & & 0.127 & 0.385 & $0.001^{8}$ & & $0.001^{2}$ & \\
\hline & M.D. & $=0.200$ & -4.060 & -0.801 & -3.201 & -4.001 & -5.801 & & -2.400 & -1.401 & $=7.801$ & -5.200 & -5.000 & -4.400 \\
\hline \multirow{2}{*}{\begin{tabular}{|l|}
$E$ \\
$15 \mu \mathrm{g}$
\end{tabular}} & Sig. & $0.019^{*}$ & 0.321 & $0.007^{*}$ & 0.649 & 0.186 & $0.041^{3}$ & 0.127 & & 0.508 & $0.001^{*}$ & 0.083 & $0.048^{*}$ & 0.215 \\
\hline & M.D. ${ }^{8=}$ & -3.801 & -1.601 & -4.333 & -0.733 & -2.133 & -3.333 & 2.466 & & 1.066 & 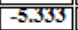 & -2.801 & -3.201 & -2.001 \\
\hline \multirow{2}{*}{$\begin{array}{c}\mathrm{K} \\
40 \mu \mathrm{g}\end{array}$} & Sig. & $0.003^{\circ}$ & 0.099 & $0.001^{\circ}$ & 0.264 & $0.048^{\circ}$ & $0.007 \%$ & 0.385 & .508 & & $0.001 \%$ & $0.017^{\circ}$ & $0.009^{\circ}$ & 0.058 \\
\hline & M.D. ${ }^{* 8 *}$ & -4.866 & -2.666 & -5.401 & -1.801 & -3.201 & -4.401 & 1.401 & -1.066 & & -6.401 & -3.866 & -4.266 & -3.066 \\
\hline & Sig. & 0.341 & $0.021^{2}$ & 0.535 & $0.004^{2}$ & $0.048^{\circ}$ & 0.215 & $0.001^{\circ}$ & $0.001^{\circ}$ & $0.001^{8}$ & & 0.117 & 0.186 & $0.014^{*}$ \\
\hline $10 \mu \mathrm{g}$ & M.D. ${ }^{\text {sष }}$ & 1.533 & 3.733 & 1.001 & 4.601 & 3.201 & 2.001 & 7.801 & 5.333 & 6.401 & & 2.533 & 2.133 & 3.333 \\
\hline \multirow{2}{*}{$\begin{array}{c}\mathrm{OA} \\
2 \mu \mathrm{g}\end{array}$} & Sig. & 0.535 & 0.456 & 0.341 & 0.201 & 0.679 & 0.741 & $0.001^{8}$ & 0.083 & $0.017^{\circ}$ & 0.117 & & 0.803 & 0.619 \\
\hline & M.D. ${ }^{87}$ & -1.001 & 1.201 & -1.533 & 2.066 & 0.666 & -0.533 & 5.266 & 2.801 & 3.866 & -2.533 & & -0.401 & 0.801 \\
\hline \multirow{2}{*}{$\begin{array}{c}\mathrm{B} \\
10 \mu \mathrm{g}\end{array}$} & Sig. & 0.709 & 0.321 & 0.482 & 0.127 & 0.508 & 0.934 & $0.001^{2}$ & $\overline{0.048^{\circ}}$ & $0.009^{\circ}$ & 0.186 & 0.803 & & 0.456 \\
\hline & M.D. ${ }^{* 8}$ & -0.600 & 1.601 & -1.133 & 2.466 & 1.066 & -0.133 & 5.666 & 3.201 & 4.266 & -2.133 & 0.401 & & 1.201 \\
\hline \multirow{2}{*}{$\begin{array}{l}C \mathrm{CC} \\
5 \mu \mathrm{g}\end{array}$} & Sig. & 0.264 & 0.803 & 0.148 & 0.432 & 0.934 & 0.408 & $0.006^{8}$ & 0.215 & 0.058 & $0.014^{8}$ & 0.619 & 0.456 & \\
\hline & M.D. & & & & & 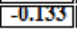 & & & & & & & & \\
\hline
\end{tabular}

*The mean difference is significant at the $\mathbf{P}$-Value $=\mathbf{0 . 0 5}$ level.

**Mean difference.

DOI: $10.9790 / 3008-1104022739$

www.iosrjournals.org

$35 \mid$ Page 
This table explained the significantly differences between different types of antibiotics when study their activities against $P$. mirabilis by statistical analysis depended on LSD system.

Also showed presences of significant differences between these antibiotics, and this explained through the star that found at p-valve equal or less than 0.05 level.

However, not all antibiotics have significant differences when comparison with each other, some of them have no significant differences, and this showed through the star that not found at p-valve at 0.05 level.

The comparisons involved two types, first the $\mathrm{X}$ side (antibiotics) comparison with $\mathrm{Y}$ side (antibiotics) and the second involved comparison the $\mathrm{Y}$ side (antibiotics) with $\mathrm{X}$ side (antibiotics).

Figure:8. Show the modes of action of different types of antibiotics against Proteus mirabilis

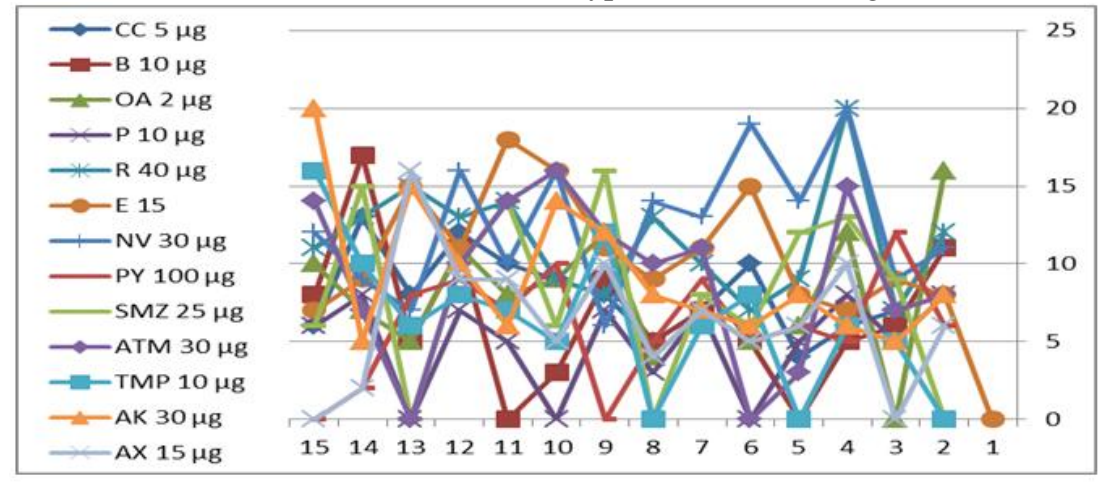

This figure explained the antibiotics types have different modes of actions, this mean each antibiotic showed inhibition zone with different size against same bacteria with several replicates (15 replicates).

Figure:9. Show the percent of action of different types of antibiotics against Proteus mirabilis

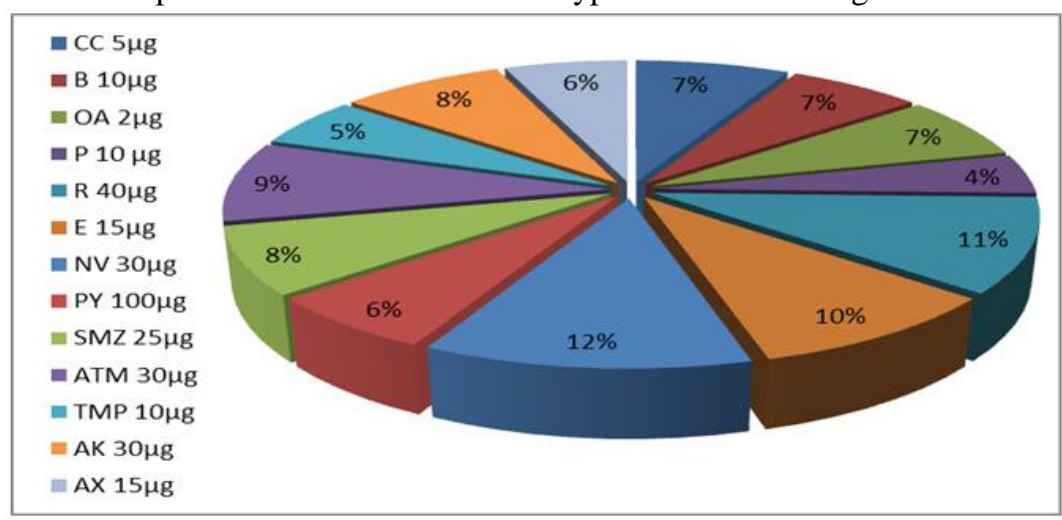

This figure showed the action percentages of different antibiotic types; also explained the NV 30 antibiotics has high percent equal to $12 \%$ while the P 10 antibiotic has low percent equal to $4 \%$ from all antibiotics activities against Proteus mirabilis.

Figure:10. Show the comparisons between all antibiotics means against pathogenic gram negative

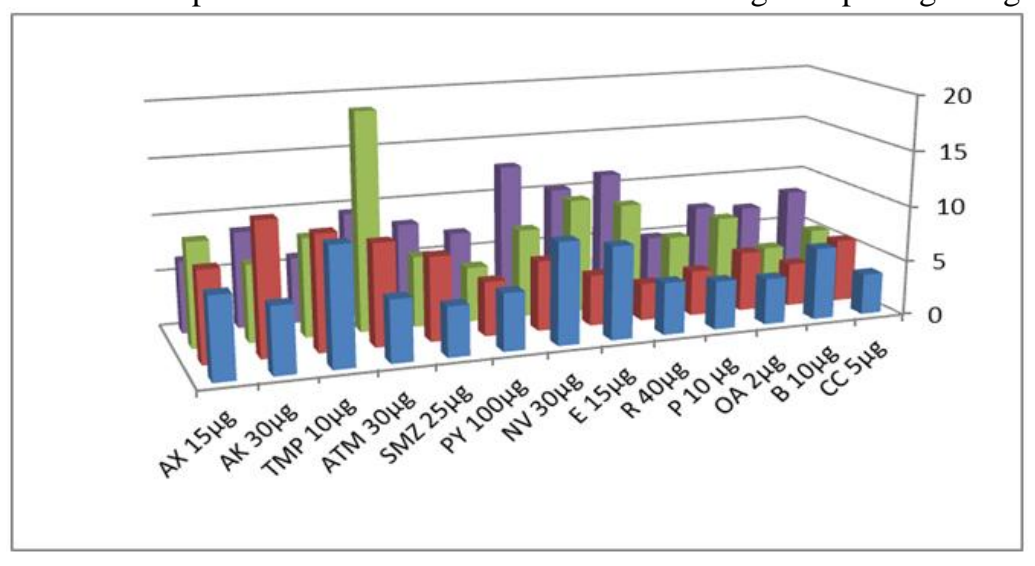


This figure showed the ATM 30 (Aztreonam30) antibiotic has high activity against in comparison with all other types of antibiotics.

\section{Discussion}

In this study showed the (Trimethoprim) antibiotic has high activity against Ps. aeruginosa (Table:3 and Figure:3.). These results were agreed with the Kamel et al who found the isolate of this bacteria were sensitive to this antibiotic (Kamel et al., 2011). While dis agreed with Rosas et al who found the isolate of this bacteria were resistant to trimethoprim (Rosas et al., 2015). The present study explained the (Clindamycin) antibiotic has low activity against Pseudomonas aeruginosa. These results were agreed with Rosas et al who found the isolate of this bacteria were resistant to this antibiotic (Rosas et al., 2015). In the current study showed the (Penicillin-G) antibiotic has low activity against Pseudomonas aeruginosa. These results were agreed with Javiya et al who found this bacteria resistant to many antibiotics such as penicillin-G (Javiya et al., 2008). Pseudomonas aeruginosa is a major causative agent for nosocomial infections in immunocompromised and burn patients in hospitalized (Biswal et al., 2014). This bacteria is have ability to resistant several types of antibiotics because production of antibiotic inactivating enzymes and low permeability of its outer membrane, this expression of various efflux pumps and the (Hillier et al., 2006).

In the present study explained the (Amikacin) antibiotic has high activity against Escherichia coli (Table:5 and Figure:5.). These results were agreed with Niranjan and Malini who found the isolates of this bacteria were sensitive to this antibiotic (Niranjan and Malini., 2014). But in study of Ruiz et al who found some isolates of this bacteria were resistant to this antibiotic (Ruiz et al., 2005). The current study explained the (Bacitracin) antibiotic has low activity against Escherichia coli. These results were agreed with Wang et al who found the isolate of this bacteria were resistant to this antibiotic (Wang et al., 2013). In the this study showed the (Rifaximin) antibiotic has low activity against Escherichia coli. These results were agreed with Kothary et al who found this bacteria resistant to this antibiotic (Kothary et al., 2013). Escherichia coli is the most common pathogens causing diarrhea with mild to severe watery diarrhea in humans of all ages (Qadri et al., 2005). The isolates of this bacteria have ability to resistant of multiple antibiotic types and this has been reported and involving four or more unrelated families of antibiotics, (Maynard et al., 2003). This resistance determinants by or mobile genetic elements including transposons, plasmids and generics transformation (Carattoli., 2001).

In the present study showed the (Aztreonam) antibiotic has high activity against Enterobacter cloacae (Table:7 and Figure:7.). These results were agreed with Igari who found this antibiotic has activity against this bacteria (Igari., 1994). But dis agreed with Giamarellou et al who found the isolate of Enterobacter cloacae were resistant to Aztreonam (Giamarellou et al., 1989). In the current study showed the (Bacitracin) antibiotic has low activity against Enterobacter cloacae. These results were agreed with Sharma et al who found the isolates of this bacteria were resistant to this antibiotic (Sharma et al., 2015). Enterobacter cloacae have been reported as important opportunistic and multidrug resistant pathogenic bacteria for humans during the last three decades in wards of hospital (Davin-Regli and Pages., 2015). E.cloacae causes a wide spectrum of infections involving the urinary tract and several part of human body (Karam and Heffer., 2000). Most isolates of the this bacteria are complex are intrinsically resistant to many types of antibiotics (Stock et al., 2001).

In this study explained the (Novobiocin) antibiotic has high activity against Proteus mirabilis (Table:9 and Figure:9.). These results were agreed with Safary et al who found this antibiotic has high activity against this bacteria (Safary et al., 2009). But in study of Al-Mutairi et al who found this antibiotic has moderate activity against this bacteria (Al-Mutairi et al., 2016). The present study showed the (Penicillin-G) antibiotic has low activity against Proteus mirabilis. These results were agreed with Stock who found the isolate of this bacteria were resistant to this antibiotic (Stock., 2003). The Proteus mirabilis are widespread in the environment and most common infections in the human including urinary tract infections (Armbruster and Mobley., 2012). Now the multidrug resistance becoming more common in this bacteria (Mokracka et al., 2012).

Figures (1), (2), (4), (6) and (8) explained the antibiotics in this study have different modes of action. These variations deepened on the bacterial types, antibiotic types, mechanisms of bacterial resistant and mode of antibiotics action. However, Antibiotics can be classified based on the system they affect or the cellular component, in addition to whether they induce cell death (bactericidal drugs) or inhibit cell growth (bacteriostatic drugs) (Kohanski et al., 2010). In general, most of antibiotics acts by one of the following, inhibit nucleic acids such as inhibit DNA and/or RNA synthesis, replication and joining, inhibit cell wall synthesis and/or breakdown, and/or protein synthesis (Walsh., 2003).

Figure (10) showed the Aztreonam has more activity in comparisons with others. Aztreonam is a bactericidal antibiotic which interferes with the synthesis of the bacterial cell wall (Georgopapadakou et al., 1982). This antibiotic has excellent activity against major gram negative pathogens with bactericidal activity (Neu., 1988). Aztrenoam has been used successfully in the treatment of both upper and lower urinary tract infections(Khanb and Shah., 2001). It is has been effective in the treatment of gram negative bacteremia (Scully and Henry., 1985). Bone and Joint Infections caused by susceptible strains of E. coli, Proteus and Pseudomonas 
have been treated with Aztreonam (Simons and Lee., 1985). It is useful in treatment of multi drug resistant Shigella and Salmonella as well as in therapy of gastro intestinal gram negative organisms (Wasfy et al., 2000). Aztreonam has been shown to penetrate inflamed meninges, and reach therapeutic levels in the central nervous system (Kapoor and Gathwala., 2004).

\section{References}

[1]. Alhazmi. A. (2015). Pseudomonas aeruginosa Pathogenesis and Pathogenic Mechanisms. International Journal of Biology. 7(2): 44-67.

[2]. Allocati, N. Masulli, M. Alexeyev, M.F. and Carmine, B.D. (2013). Escherichia coli in Europe: An Overview. Int. J. Environ. Res. Public Health. 10: 6235-6254.

[3]. Al-Mutairi, M.H. Ali, S. Aly, S.M. and Aldebasi, Y. (2016). Antimicrobial activity of Sider (Ziziphus Spina- Christi), Leaves extract against selected pathogenic bacteria. Ejpmr. 3(5): 138-144.

[4]. Anuj Nautiyal, N.V. Madhav, S. Sharma R.K. Ojha, A. and Bhargava, S. (2015). Review on Nosocomial Infections. Carib. J .Sci Tech. 3: 781-788.

[5]. Armbruster, C.E. and Mobley, H.L.T. (2012). Merging mythology and morphology: the multifaceted lifestyle of Proteus mirabilis. Nat Rev Micro. 10: 743-754.

[6]. Azimi, A. Naserpour., T. Bazmi., F. Peymani., A. Aslanimehr., M. Saadat, S. (2015). Evaluation of oprD Gene Expression in Carbapenem-Resistant Pseudomonas aeruginosa Strains Isolated From Severe Burn Patients With Secondary Infection. Biotech Health Sci. 2(3): 43- 47.

[7]. Bahashwan, S.A. (2013). Antimicrobial Resistance Patterns of Proteus isolate from clinical specimens. European Scientific Journal September. 9(27): 1857-7881.

[8]. Bauer, A.W. Kirby, W.M.M. Sherris, J.C. and Turck, M. (1966). Antibiotic susceptibility testing by a standardized single disk method. Am. J. Clin. Pathol., 45: 493496.

[9]. Biswal, I. Arora, B.S. Kasana, D. (2014). Incidence of multidrug resistant pseudomonas aeruginosa isolated from burn patients and environment of teaching institution. J Clin Diagn Res. 8(5): 26-29.

[10]. Carattoli, A. (2001). Importance of integrons in the diffusion of resistance. Vet. Res. 32: 243-259.

[11]. Cheesbrough, M. (2006). District Laboratory Practice in Tropical Countries. Part 2. Cambridge University Press, Cambridge, 434.

[12]. Dadheech, T. Vyas, R. and Rastogi, V. (2015). Antibiotics Resistance of aerobic bacterial isolates of Proteus mirabilis from sick layer Chickens infected with septicemia and salinities in Ajmer region of Rajasthan. World Journal of Pharmacy and Pharmaceutical Sciences. 4(7): 2002-2011.

[13]. Davies, J. and Davies., D. (2010). Origins and Evolution of Antibiotic Resistance. Microbiology and Molecular Biology Rev. (74)3: 417-433.

[14]. Davin-Regli, A. and Pages, J. M. (2015). Enterobacte raerogenes and Enterobacter cloacae; versatile bacterial pathogens confronting antibiotic treatment. FrontiersinMicrobiology. 6: 1-10.

[15]. Eguchi, H. Miyamoto, T. Kuwahara, T. Mitamura, S. and Mitamura, Y. (2013). Infectious conjunctivitis caused by Pseudomonas aeruginosa isolated from a bathroom. BMC Res Notes. 6:245. doi:10.1186/1756-0500-6-245.

[16]. Ewers, C. Bethe, A. Semmler, T.Guenther, S. and Wieler, L.H. (2012). Extended-spectrum beta-lactamase-producing and AmpCproducing Escherichia coli from livestock and companion animals, and their putative impact on public health: A global perspective. Clin. Microbiol. Infect. 18: 646-655.

[17]. Feglo, P.K. Gbedema, SY. Quay, S.N.A. Adu-Sarcode, Y.O. poku, O. and krah,C. (2010). Occurrence species distribution and antibiotic resistance of Proteus isolates: A case study at the Komfo Anoke Teaching Hospital(KATH) in Ghana. J.International Journal of Pharma science and research.1(9): 347-352.

[18]. Fisher, J.F. and Mobashery., S. (2010). Enzymology of Bacterial Resistance. Comprehensive Natural Products II. In: Enzymes and Enzyme Mechanisms. Elsevier. 8: 443-487.

[19]. Gellatly, S.L. and Hancock, R.E.W. (2013). Pseudomonas aeruginosa: new insights into pathogenesis and host defenses. Pathog Dis. 67:159-173.

[20]. Georgopapadakou, N.H. Smit, S.A. and Sykes, R.B. (1982). Modes of action of Aztreonam. Antimicrobial Agents Chemother. 21: 950-956.

[21]. Giamarellou, H. Evagelopoulou, V. and Lelekis, M. (1989). Enhanced in-vitro bactericidal activity of amikacin combined with latamoxef, cefotaxime and aztreonam against multi-resistant Enterobacter cloacae. Journal of Antimicrobial Chemotherapy. 23(4): $537-45$.

[22]. Hara, C.M. Brenner, F.W. and Miller, J.M. (2000). Classification, identification, and clinical significance of Proteus, Providencia, and Morganella . Clin Microbiol Rev.13(4): 534-546.

[23]. Hillier, A. Alcorn, J.R. Cole, L.K. Kowalski, J.J. (2006) Pyoderma caused by Pseudomonas aeruginosa infection in dogs: 20 cases. Vet Dermatol. 17(6): 432-439.

[24]. Holt, J.G. N.R. Krieg, P.H.A. Sneath, J.T. Staley, and S.T. Williams. (1994). Bergy's manual of determinative bacteriology. 9th ed. Williams and Wilkins, Baltimore, USA.

[25]. Igari, J. (1994). Antibacterial activity of aztreonam against clinical isolates. Jpn J Antibiot. 47(5):502-520.

[26]. Jabalameli, F. Mirsalehian, A. Sotoudeh, N. Jabalameli, L. Aligholi, M. and Khoramian., B. (2011). Multiple-locus variable number of tandem repeats (VNTR) fingerprinting (MLVF) and antibacterial resistance profiles of extended spectrum beta lactamase (ESBL) producing Pseudomonas aeruginosa among burnt patients in Tehran. Burns. 37(7): 1202-1207.

[27]. Javiya, V.A. Ghatak, S.B. Patel, K.R. \& Patel, J.A. (2008). Antibiotic susceptibility patterns of Pseudomonas aeruginosa at a tertiary care hospital in Gujarat, India. Indian Jourl of Pharma. 40(5): 230-234.

[28]. Johnson, T.J. Logue, C.M. Johnson, J.R. Kuskowski, M.A. Sherwood, J.S. Barnes, H.J. DebRoy, C. Wannemuehler, Y.M. ObataYasuoka, M. and Spanjaard, L. (2012). Associations between multidrug resistance, plasmid content, and virulence potential among extra intestinal pathogenic and commensal Escherichia coli from humans and poultry. Foodborne Pathog. rrDis. 9: 37-46.

[29]. Kamel, G.M. Ezz eldeen, N.A. El-Mishad, M.Y. and Ezzat,R.F. (2011). Susceptibility Pattern of Pseudomonas aeruginosa Against Antimicrobial Agents and Some Plant Extracts with Focus on its Prevalence in Different Sources. Global Veterinaria. 6 (1): 61-72.

[30]. Kapoor,S. and Gathwala, G. (2004). Aztreonam. Drug Therapy. 41(17): 359-364.

[31]. Karam, G.H. and Heffer. J.E. (2000). Emerging issue in Antibiotic Resistance in Blood - borne Infections. Am. J. Respir. Crit. Care. Med. 162(5): 1610-1616. 
[32]. Khan, M.T. and Shah, S.H. (2001). Comparison of aztreonam against other antibiotics used in urinary tract infections. $J$ Ayub Med Coll Abbottabad. 13: 22-24.

[33]. Kohanski, M.A. Dwyer, D.J. and Collins, J.J (2010). How antibiotics kill bacteria: from targets to networks. Nat Rev Microbiol. 8(6): 423-435.

[34]. Kothary, V. Scherl, E.J. Bosworth, B. Jiang, Z.D. DuPont, H.L. Harel, J. W. Simpson, K.W and Dogane, B. (2013). Rifaximin Resistance in Escherichia coli Associated with Inflammatory Bowel Disease Correlates with Prior Rifaximin Use, Mutations in rpoB, and Activity of Phe-Arg- $\beta$-Naphthylamide-Inhibitable Efflux Pumps. Antimicrobial Agents and Chemotherapy.57(2) : 811817.

[35]. Manikandan, C. and Amsath, A. (2014). Antibiotic susceptibility pattern of Escherichia coli isolated from urine samples in Pattukkottai, Tamilnadu. Int.J.Curr.Microbiol.App.Sci. 3(10): 449-457.

[36]. Maynard, C., Fairbrother, J.M. Bekal, S. Sanschagrin, F. Levesque, R.C. Brousseau, R. Masson, L. Lariviere, S. and Harel, J. (2003). Antimicrobial resistance genes in enterotoxigenic Escherichia coli O149:K91 isolates obtained over a 23-year period from pigs. Antimicrob. Agents Chemother. 47:3214-3221.

[37]. Mezzatesta, M.L. Gona, F. and Stefani, S. (2012). Enterobactercloacae complex: clinical impact and emerging antibiotic resistance. Future Microbiol. 7: 887-902.

[38]. Mirsalehian, A. Feizabadi., M. Nakhjavani., F.A. Jabalameli., F. Goli, H. and Kalantari., N (2010). Detection of VEB-1, OXA-10 and PER-1 genotypes in extended-spectrum beta-lactamase-producing Pseudomonas aeruginosa strains isolated from burn patients. Burns. 36(1): 70-4.

[39]. Mokracka, J. Gruszczynska, B. and Kaznowski, A. (2012). Integrons, $\beta$-lactamase and qnr genes in multidrug resistant clinical isolates of Proteus mirabilis and P. vulgaris. APMIS 2012;120: 950-958.

[40]. Murray, J.L. Kwon, T. Marcotte, E.M. and Whiteleya, M. (2015). Intrinsic Antimicrobial Resistance Determinants in the Superbug Pseudomonas aeruginosa. mbio.asm.org. 6(6): 1-10.

[41]. Nabeela, N. Munazza, A. Shekh., A.R. and Zaid, A.P. (2004). Urinary tract infections associated with multidrug resistant enteric bacilli: characterization and genetical studies. Journal of Pakistan Pharma. Scien. 17(2): 115-123.

[42]. Neu H.C. (1988). Aztreonam: The first monobactam. Med Clin North Am. 72: 556-566.

[43]. Niranjan, V. and Malini, A. (2014). Antimicrobial resistance pattern in Escherichia coli causing urinary tract infection among inpatients. Indian J Med Res. 139: 945-948

[44]. Otoikhian, C.S.O. and Tanimowo, W.O. (2016) Identification of intestinal microbes of pig sold in local market in Niger delta environment. Nigerian Journal of Agriculture, Food and Environment. 12(1): 111-115.

[45]. Pelczar, M. Reid, R. and Chan, E. (1957). Microbiology. T.M.H. Edition, Tata McGraw-Hill Publishing Company Ltd.,Noida

[46]. Penner, J.L. (1984). Genus XI.Proteus , Bergey's manual of systemic bacteriology. In N.R. Krieg and J.G. Holt(ed.),The William and Wilkins Co., Baltimore, Md. .1: 491-494.

[47]. Perez, A. Poza, M. Aranda, J. Latasa, C. Medrano, F.J. and Tomas, M. (2012). Effect of transcriptional activators SoxS, RobA, and RamA on expression of multidrug efflux pump AcrAB-TolC in Enterobacter cloacae. Antimicrob. Agents Chemother. 56, 62566266.

[48]. Pestourie, N. Garnier, F. Barraud,O. Bedu, A. Ploy, M. C. and Mounier, M.(2014). Outbreak of AmpC $\beta$-lactamase hyper producing Enterobacter cloacae in a neonatal intensive care unit in a French teaching hospital. Am. J. Infect. Control. 42: 456-458.

[49]. Pitout, J. D. (2012). Extraintestinal pathogenic Escherichia coli: An update on antimicrobial resistance, laboratory diagnosis and treatment. Expert Rev. Anti Infect. Ther. 10: 1165-1176.

[50]. Qadri, F. Svennerholm, A.M. Faruque, A.S. and Sack, R.B.(2005). Enterotoxigenic Escherichia coli in developing countries: Epidemiology, microbiology, clinical features, treatment, and prevention. Clin. Microbiol. Rev. 18: 465-483.

[51]. Rosas, Y.B. Munoz, K.O. and Garcia O.T. (2015). Pseudomonas aeruginosa: an emerging nosocomial trouble in veterinary; Pseudomonas aeruginosa: un problema nosocomial emergente en veterinaria. Rev.MVZ Cordoba. 20 :4937-4946.

[52]. Ruiz, J. Bertran, S. Sauca, G. Julia, A. Vila, X. Gomez, E. Jimenez de Anta, M. T and Vila, J. (2005). Isolation of an amikacin resistant Escherichia coli strain after tobramycin treatment of previous recurrent episodes of respiratory tract infections caused by Pseudomonas aeruginosa. Clin Microbiol Infect. 11: 71-73.

[53]. Safary, A. Motamedi, H. Maleki, S. and Seyyednejad, S.M. (2009). A Preliminary Study on the Antibacterial Activity of Quercus brantii Against Bacterial Pathogens, Particularly Enteric Pathogens. IJB, 5: 176-180.

[54]. Scully, B.E. and Henry, S.A. (1985). Clinical experience with Aztreonam in the treatment of gram negative bacterimia. Rev Infect Dis. 7: S 789-793.

[55]. Sharma, S.K. Rahul Yadav, R. and Nathawat, P. (2015). Multidrug Resistance Pattern of Enterobacter Spp. Isolated from Acute Respiratory Tract Infected Camels (Camelus dromedarius). Adv. Anim. Vet. Sci. 3(2): 128-132.

[56]. Simons W.J. and Lee, T.J. (1985). Aztreonam in the treatment of bone and joint infections caused by gram negative bacilli. Rev Infect Dis. 7: 783-788.

[57]. Singla, P. Sangwan, J. Garg, S. and Chaudhary, U. (2015). Prevalence and Antibiogram of Multidrug resistant Uropathogenic Isolates of Proteus mirabilis in a Teaching Tertiary Care Hospital. Int.J.Curr.Microbiol.App.Sci. 4(12): 675-682.

[58]. Stiles, M and LAI, K.N.G. (1981). Biochemical Characteristics and Identification of Enterobacteriaceae Isolated from Meats. Appl. and Envir. Microbio. Mar. Vol. (41) 3: 639-645.

[59]. Stock, I. Gruger, T. Wiedemann, B. (2001). Natural antibiotic susceptibility of strains of the Enterobacter cloacae complex. Int. J. Antimicrob. Agents.18 (6): 537-545.

[60]. Stock, I. (2003). Natural antibiotic susceptibility of Proteus spp., with special reference to P. mirabilis and P. penneri strains. $J$ Chemo ther. 15(1): 12-26.

[61]. Strateva, T. and Yordanov, D. (2009). Pseudomonas aeruginosa-a phenomenon of bacterial resistance. J Med Microbiol. 58(9): 1133-1148.

[62]. Walsh. C. (2003). Antibiotics: actions, origins, resistance. ASM Press; Washington, D.C

[63]. Wang, D. Hu, E. Chen, J. Tao, X. Gutierrez, K. and Qi, Y. (2013). Characterization of novel ybjG and dacC variants in Escherichia coli. Journal of Medical Microbiology. 62: 1728-1734.

[64]. Wasfy, M.O. Oyofo, B.A. David, J.C. Ismail, T.F. El-Gendy, A.M. and Mohran, Z.S. (2000). Isolation and antibiotic susceptibility of Salmonella, Shigella and Campylobacter from acute enteric infections in Egypt. J Health Popul Nutr. 18: 33-38.

[65]. Wong, M.H. Wan, H.Y. and Chen, S. (2013). Characterization of multidrug-resistant Proteus mirabilis isolated from chicken carcasses. Foodborne Pathog Dis. 10: 177-181.

[66]. Yoo, S. H. Jeong, H. Kwon, S.K. and Kim, J.F. (2009). Genomics, Biological Features, and Biotechnological Applications of Escherichia coli B: Is $B$ for better; Springer: Berlin, Germany. 